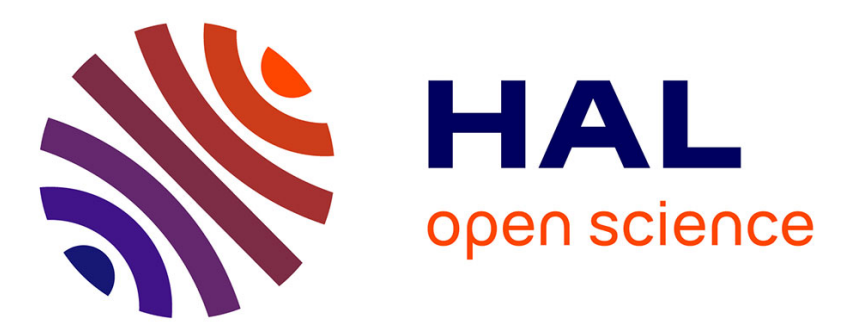

\title{
Stochastic modelling of three-dimensional particle rebound from isotropic rough wall surface
}

\author{
Darko Radenkovic, Olivier Simonin
}

\section{To cite this version:}

Darko Radenkovic, Olivier Simonin. Stochastic modelling of three-dimensional particle rebound from isotropic rough wall surface. International Journal of Multiphase Flow, 2018, 109, pp.35-50. 10.1016/j.ijmultiphaseflow.2018.07.013 . hal-02135779

\section{HAL Id: hal-02135779 \\ https://hal.science/hal-02135779}

Submitted on 21 May 2019

HAL is a multi-disciplinary open access archive for the deposit and dissemination of scientific research documents, whether they are published or not. The documents may come from teaching and research institutions in France or abroad, or from public or private research centers.
L'archive ouverte pluridisciplinaire HAL, est destinée au dépôt et à la diffusion de documents scientifiques de niveau recherche, publiés ou non, émanant des établissements d'enseignement et de recherche français ou étrangers, des laboratoires publics ou privés. 


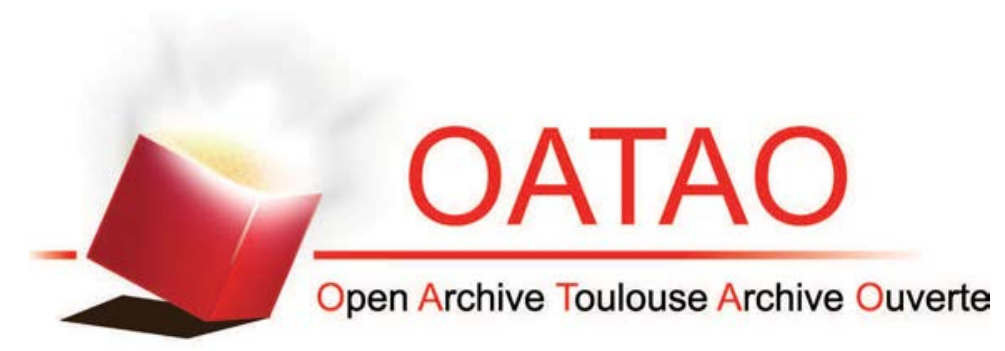

\section{Open Archive Toulouse Archive Ouverte (OATAO)}

OATAO is an open access repository that collects the work of some Toulouse researchers and makes it freely available over the web where possible.

This is an author's version published in: https://oatao.univ-toulouse.fr/23411

Official URL : https://doi.org/10.1016/j.ijmultiphaseflow.2018.07.013

\section{To cite this version :}

Radenkovic, Darko and Simonin, Olivier Stochastic modelling of three-dimensional particle rebound from isotropic rough wall surface. (2018) International Journal of Multiphase Flow, 109. 35-50. ISSN 0301-9322

Any correspondence concerning this service should be sent to the repository administrator: tech-oatao@listes-diff.inp-toulouse.fr 


\title{
Stochastic modelling of three-dimensional particle rebound from isotropic rough wall surface
}

\author{
Darko Radenkovic ${ }^{\mathrm{a}, *}$, Olivier Simonin ${ }^{\mathrm{b}}$ \\ a Faculty of Mechanical Engineering, University of Belgrade, Belgrade, Serbia \\ ${ }^{\mathrm{b}}$ Institut de Mécanique des Fluides (IMFT), Université de Toulouse, CNRS, Toulouse, France
}

Keywords:

Three-dimensional rough wall

Stochastic modelling

Deterministic simulations

Particle-wall interaction

Shadow effect model

Multiple particle rebounds

\begin{abstract}
A B S T R A C T
This paper describes an extension of the two-dimensional approach to particle-rough wall collision modelling (Sommerfeld and Huber, 1999; Konan et al., 2009) to the case of three-dimensional particle rebound from an isotropic rough wall surface. The virtual three-dimensional rough wall is represented as a Gaussian correlated surface. Normal vector angle statistical distributions are investigated in detail for such virtual rough walls, and a statistical modelling approach for these angles is proposed and validated in the frame of the low roughness approximation. Next, deterministic simulations of fully elastic particle collisions with the three-dimensional virtual wall roughness structure are carried out for various particle incident angles. It is shown that the rebound angle, in the bouncing plane of the particle, obeys the distribution given by the two-dimensional modelling approach. However, the three-dimensional structure induces a transverse deviation bouncing angle that obeys a Gaussian distribution with a standard deviation that increases with increase in incident angle. A statistical modelling approach for the virtual wall normal vector seen by any particle for a given incident angle is proposed and validated from deterministic simulation results. The probability that particles make only one rebound is in agreement with the two-dimensional multiple-collision model assumption. A new stochastic procedure for particle-isotropic rough wall interactions in a Lagrangian framework is developed and verified by comparisons with deterministic simulations and available experimental results.
\end{abstract}

\section{Introduction}

Particle-laden wall-bounded turbulent flows have wide industrial applications and for that reason they have been studied extensively using both numerical and experimental approaches. These flows are highly complex in space and time and many mechanisms are often coupled. Experimental studies (Sommerfeld and Kussin, 2004; Benson et al., 2005) and numerical studies (Tsuji et al., 1987; Sommerfeld, 2003; Squires and Simonin, 2006; Vreman, 2007; Konan et al., 2011; Breuer et al., 2012; Malloupas and van Wachem, 2013; Vreman, 2015) have indicated that the wall roughness can significantly influence both particle and fluid flows. The main effect of wall roughness in confined particle-laden flows is re-dispersion of particles by amplification of the particle wall-normal velocity component. This leads to more uniform particle profiles, a higher wall collision frequency and consequently a greater pressure drop in particle-laden flow than in single-phase flow, increasing with solid mass loading.

\footnotetext{
* Corresponding author.

E-mail address: dradenkovic@mas.bg.ac.rs (D. Radenkovic).
}

In the particle-rough wall modelling approach, the actual rough wall is often replaced with a virtual wall (see, for example, Sommerfeld and Huber, 1992). In such an approach, the computation of the interaction between a spherical particle and a true rough surface is replaced with an equivalent interaction between the particle centre and an effective virtual wall.

Deterministic and stochastic modelling approaches may be differentiated according to the method used for prediction of the wall inclination seen by incident particles (see Fig. 1). In the deterministic wall modelling approach, a rough wall surface is generated beforehand and the wall inclination seen by any incident particle is calculated when the distance between the particle centre and the rough surface is equal to the particle radius (Vreman, 2015) or less than the particle radius (De Marchis et al., 2016; Milici, 2018). In deterministic simulations whose results are shown in this paper wall inclination is calculated when particle centre intersects virtual wall which is identical to the case where the distance between particle centre and true rough surface is equal to particle radius. In the stochastic modelling approach, when the particle centre reaches particle radius distance from a certain limit boundary, a virtual wall inclination is generated according to a random process 


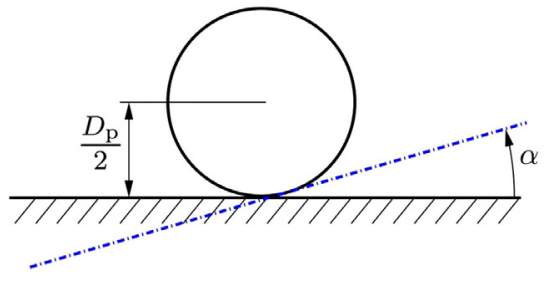

(a) Stochastic approach

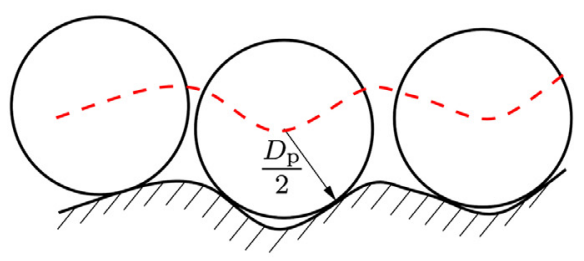

(b) Deterministic approach

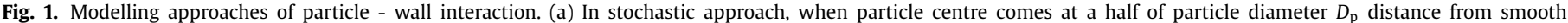

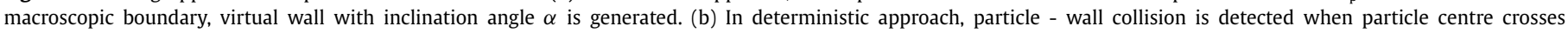

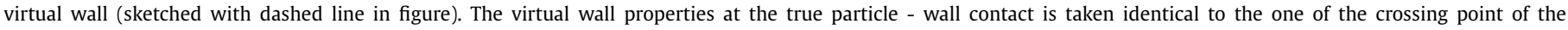
particle centre. The sketched roughness height is exaggerated with respect to the particle diameter.

following a given probability distribution dependent on the particle incident angles. Deterministic models are more computationally expensive owing to the need to find the exact point of impact of the particle surface on the rough wall (or in the case of virtual wall generation, the exact point of impact of the particle centre on the virtual wall) and, in practical Lagrangian simulations of particleladen flows, stochastic modelling approaches are preferred.

The virtual wall concept was introduced by Tsuji et al. (1985) and subsequently redefined (Tsuji et al., 1987). In these models, parameters were empirical, determined by comparisons between experimental and numerical studies in a horizontal pipe. The virtual wall was introduced for incident particle angles less than $7^{\circ}$. For larger incident angles, the virtual wall was not introduced, assuming that after collision of the particles with the flat wall, the particles returned into the flow with a significant wall normal component. However, more correct particle rebound would be calculated if a virtual wall was introduced for all particle incident angles. The reader is referred to Konan et al. (2009) for a detailed review of other virtual wall concepts.

For most surfaces in engineering practice, virtual wall roughness inclinations may be represented using Gaussian distributions, with zero mean and standard deviations $\Delta \gamma$ that depend on the wall structure and the particle diameter (Sommerfeld and $\mathrm{Hu}-$ ber, 1992; 1995; Konan et al., 2009). However, owing to the incident perspective, particles at low incident angles do not see the lee side of roughness with the same probability as the luv side of the roughness. This is called the shadow effect and was originally introduced by Sommerfeld and Huber (1999). Following Sommerfeld and Huber (1999), the inclination angle of a virtual wall can be sampled from a given modified Gaussian distribution (here referred to as the effective Sommerfeld distribution) or using a simplified procedure to account for the shadow effect (here referred to as the shadow effect model). In the shadow effect model, the virtual wall angle is sampled from a truncated Gaussian distribution to ensure that the particle incidence towards the virtual wall is realizable. Finally, in both approaches, if a particle does not return to the flow after collision with the wall, virtual wall angle sampling and particle-wall collision computation are repeated.

With an effective Sommerfeld distribution or by using the shadow effect model, a large number of grazing particles were predicted corresponding to particles with small incident angle. This was explained by the fact that these models did not account for the multiple rebounds (Konan et al., 2007) that may occur if the particle is bouncing with a small positive angle so that the particle collides with another asperity in the wall region before returning to the flow.

Konan et al. (2009) computed in a deterministic simulation the probability that a particle with a small bouncing angle will have a second collision with the wall before leaving the wall region. An analytical function depending only on the standard deviation of the wall roughness angle was proposed for this probability distribution. Finally, multiple rebounds were modelled using the stochastic approach, allowing an effective method in the Lagrangian framework called the rough wall multiple-collision model to be proposed.

Vreman (2007) carried out a DNS-DPS study of gas-particle flow through a vertical pipe with modelled particle-rough wall interaction. The virtual wall normal vector was calculated as $\mathbf{n}+\chi \mathbf{s} / \| \mathbf{n}+$ $\chi \mathbf{s} \|$, where projections of vector $\mathbf{s}$ were uniform random values from $-1 / \sqrt{3}$ to $1 / \sqrt{3}(\|\mathbf{s}\|<1)$ and the specularity coefficient $\chi=0.2$ was roughly estimated for the aluminum pipe used in the experiment, since roughness was not measured in the original experiment. It was shown that the rough wall model resulted in better agreement with experimental results than was the case without wall roughness.

Squires and Simonin (2006) studied the influence of wall roughness on the disperse phase in a vertical channel gas-solid flow. LES-DPS was applied with one-way coupling and without inter-particle collisions. The virtual wall was defined from the normal surface vector $\mathbf{n}=[\sin (\phi) \cos (\theta), \cos (\phi), \sin (\phi) \sin (\theta)]$, where $\phi$ and $\theta$ had a Gaussian distribution with standard deviation $\Delta \gamma$. After each particle-wall impact calculation, if the rebound wall-normal velocity component was not directed into the flow, angle sampling was repeated. Particle wall bouncing was calculated as frictionless.

Konan et al. (2011) compared the influences of the shadow effect model and rough wall multiple-collision model on the particle phase properties from detached eddy simulations of particle-laden flow in a horizontal rough wall channel. The effects of wall roughness were less pronounced in the case of the shadow effect model owing to the large number of grazing particles generated which led to weaker particle vertical dispersion. This problem was efficiently solved with the rough wall multiple-collision model.

Breuer et al. (2012) proposed a model for particle-rough wall interaction in which the actual wall surface was replaced with a surface covered by mono-sized spheres that represented simplified sand-grain roughness. The standard deviation of the wall roughness angle was determined from the mean roughness height $R_{z}$ (or RMS roughness $R_{q}$ ) and particle diameter. This choice of parameters permits easier application of the model in practical engineering problems. Shadow effects were modelled, but multiple rebounds were omitted. The model was applied in their LES-DPS of horizontal particle-laden channel flow.

Malloupas and van Wachem (2013) created a model for particle-wall interactions in a soft-sphere framework and verified it in an LES-DPS of horizontal particle-laden channel flow. Multiple particle rebounds were treated with successive addition of 
virtual walls. The first virtual wall was generated, with respect to the shadow effect model when a particle reached the wall roughness amplitude added to the smooth wall. If after impact with that virtual wall the particle moved closer to the wall, another virtual wall was generated and the impact was again calculated. This mechanism was repeated until the particle left the wall region.

Cheng and Zhu (2015) investigated particle-wall collisions in a connected system of virtual wall cells called a virtual-wall-group. It was shown that an effective Sommerfeld distribution was a simplified case of their new probability density function (PDF) for wall roughness angles. A distinction was made between particles that had positive and negative rebound angles. The rough wall multiple-collision procedure of Konan et al. (2009) was modified and the resulting procedure was applied in RANS-DPS simulation of particle-gas flow in a confined planar jet.

Vreman (2015) performed DNS-DPS in a vertical downward gassolid channel flow. Particle-rough wall collisions were calculated in a deterministic manner with the wall roughness modelled as densely packed half-spheres with the flat sides fixed to a smooth wall. It was reported that the wall roughness enhanced turbulence attenuation, although the diameters of the half-spheres were smaller than the viscous wall unit. The non-uniform part of mean force that free particles exerted on the gas phase, the so-called two-way coupling effect, was found to influence turbulence attenuation significantly.

The aim of this study is to investigate particle-wall interactions in a three-dimensional (3D) framework, for an isotropic rough surface, as natural extensions of the work of Sommerfeld and Huber (1999) and Konan et al. (2009) in order to provide a greater level of detail for particle-rough wall interactions, especially regarding transverse rebound characteristics.

The paper is organized as follows. In Section 2, the generation of the isotropic virtual rough wall surface is described and the angles of the normal vector to the virtual wall surface are examined in a 3D frame and statistically modelled. In Section 3, the procedure for the deterministic simulation of particle 3D rough fully elastic rebound is outlined and the results of this simulation are described. The statistical model for 3D particle collision with an isotropic rough virtual wall is proposed and verified in Section 4. In Section 5, this 3D stochastic model is applied to the particles undergoing rough wall inelastic frictional collisions. Obtained results are compared with deterministic simulation results, the twodimensional (2D) stochastic model predictions, and available experimental data.

\section{Generation and properties of the virtual rough wall surface}

\subsection{Description of Gaussian random rough surface generation}

A virtual 3D rough wall (see Fig. 2) is created according to the procedure of Garcia and Stoll (1984), as implemented by Bergström (2012). In this procedure, a Gaussian correlated surface, which here represents a virtual rough wall, is obtained in terms of RMS roughness height $h$ (in the $y$ direction) and correlation length scales in the $x$ and $z$ directions, $c_{\mathrm{L} x}$ and $c_{\mathrm{L} z}$, respectively. In the case of isotropic surfaces, studied in this work, the correlation length scales in the $x$ and $z$ directions are identical $c_{\mathrm{L} x}=c_{\mathrm{L} z}=c_{\mathrm{L}}$.

The first step in creating the rough surface is the generation of uncorrelated Gaussian random numbers $y_{\mathrm{u}}(x, z)$ with zero mean and standard deviation $h$ on the mesh in the $x-z$ plane. Correlated rough wall surface coordinates are then obtained by convolution with a spatial filter as:

$y(x, z)=\int_{-\infty}^{+\infty} \int_{-\infty}^{+\infty} f\left(x-x^{\prime}, z-z^{\prime}\right) y_{\mathrm{u}}\left(x^{\prime}, z^{\prime}\right) \mathrm{d} x^{\prime} \mathrm{d} z^{\prime}$

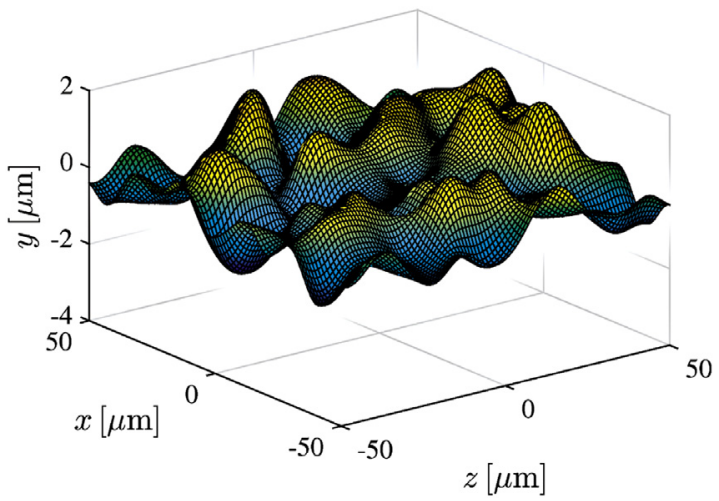

Fig. 2. Virtual rough wall with RMS roughness height $h=0.63 \mu \mathrm{m}$ and correlation length scale $c_{\mathrm{L}}=10 \mu \mathrm{m}$. The mesh resolution is $\delta x=\delta z=1 \mu \mathrm{m}$.

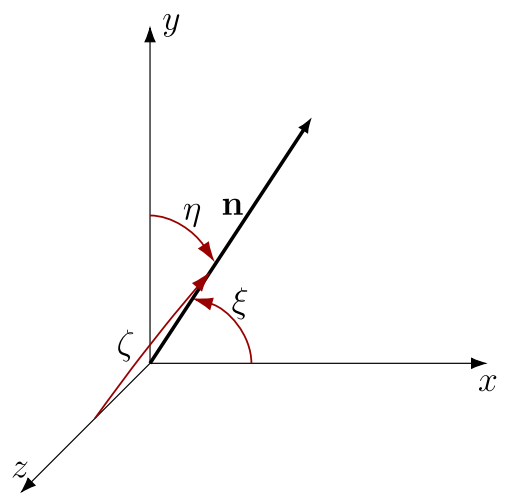

Fig. 3. Angles of the virtual wall normal vector with the coordinate axes.

where

$f(x, z)=\frac{2}{\sqrt{\pi} c_{\mathrm{L}}} \exp \frac{-2\left(x^{2}+z^{2}\right)}{c_{\mathrm{L}}^{2}}$

represents a Gaussian filter for isotropic surface generation. The Eq. (1) is calculated with the fast Fourier transform algorithm (FFT). Standard deviation of filtered roughness $y(x, z)$ is the same as the standard deviation of Gaussian random numbers $y_{\mathrm{u}}(x, z)$.

In the deterministic simulation, the particle-wall collision is detected when a particle centre trajectory crosses the virtual rough wall surface through an elementary triangular cell. Unit vectors can then be formed along the two sides of that cell in the $y-x$ and $y-z$ planes, and their vector product gives the normal surface vector directed towards the flow.

\subsection{Characterization of the 3D virtual rough wall normal vector}

Let us define $(\xi, \eta, \zeta) \in[0, \pi]^{3}$, vector angles between the virtual rough wall normal vector $\mathbf{n}$ and the unit vectors along the $x$, $y$ and $z$ axes, respectively, as shown in Fig. 3.

As shown in Fig. 3, projections of the virtual normal vector $\mathbf{n}$ are defined with,

$n_{x}=\cos (\xi), \quad n_{y}=\cos (\eta), \quad n_{z}=\cos (\zeta), \quad(\xi, \eta, \zeta) \in[0, \pi]^{3}$

so that the virtual wall normal unit vector $\mathbf{n}$ may be written as,

$\mathbf{n}=\cos (\xi) \mathbf{i}+\cos (\eta) \mathbf{j}+\cos (\zeta) \mathbf{k}$

where $\mathbf{i}, \mathbf{j}$ and $\mathbf{k}$ are the orthogonal unit vectors in the direction of the $x, y$ and $z$ axes, respectively. Scatter plots of virtual wall normal vector angles $(\eta, \xi),(\eta, \zeta)$ and $(\zeta, \xi)$ are shown in Fig. 4, from 


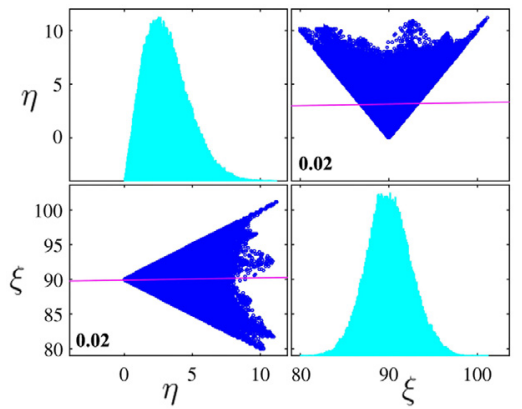

(a) Scatter plot $\eta-\xi$

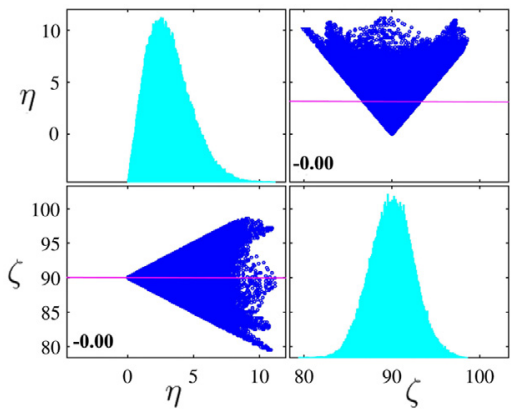

(b) Scatter plot $\eta-\zeta$

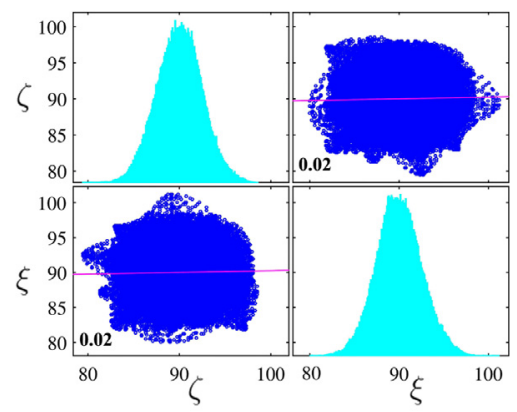

(c) Scatter plot $\zeta-\xi$

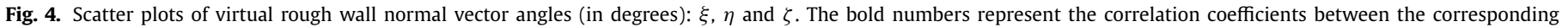

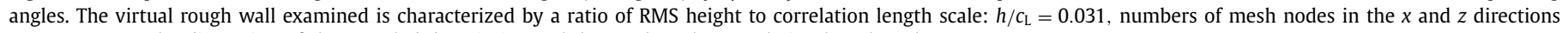
$N_{x}=N_{z}=500$. The dimension of the sampled domain is much larger than the correlation length scale $c_{\mathrm{L}}$.

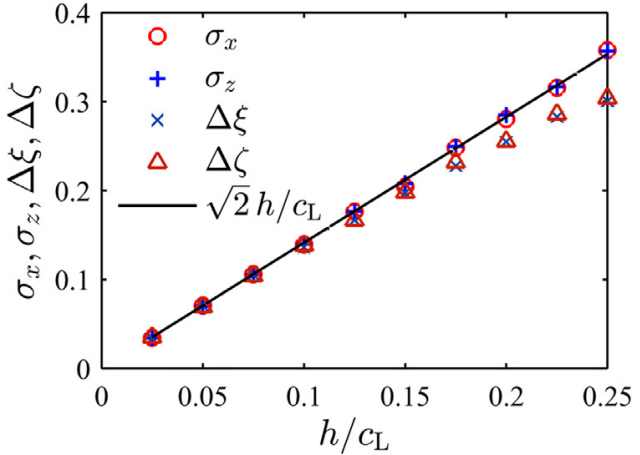

Fig. 5. Dependence of the RMS virtual wall slope in the $x$ and $z$ directions, $\sigma_{x}$ and $\sigma_{z}$, respectively, and virtual wall normal vector standard deviations $\Delta \xi$ and $\Delta \zeta$, on the ratio of the RMS roughness height $h$ to the correlation length scale $c_{\mathrm{L}}$ of the virtual rough wall. The virtual rough walls examined have $N_{x}=N_{z}=500$ nodes. The sampled domain is much larger than the correlation length scale $c_{\mathrm{L}}$.

angles $\xi, \eta$ and $\zeta$ calculated in every triangular cell of the virtual wall. The corresponding distributions show that angles $\xi$ and $\zeta$ obey two uncorrelated Gaussian distributions, with mean values $\bar{\xi}=\bar{\zeta}=\pi / 2$.

From Tsang et al. (2000), it is known that for a one-dimensional Gaussian roughness profile it holds that,

$\sigma=\sqrt{2} \frac{h}{c_{\mathrm{L}}}$

where $\sigma$ is the RMS value of the wall roughness slope.

In Fig. 5, (5) is compared with the RMS of the virtual wall slopes along the $x$ and $z$ directions, where these slopes $s_{x}=-n_{x} / n_{y}$ and $s_{z}=-n_{z} / n_{y}$, respectively, are calculated in every triangular cell and projections of the virtual normal vector $\mathbf{n}$ are defined in (3). The agreement is very good for all values compared.

The angle $\eta$ can be directly computed in terms of angles $\xi$ and $\zeta:$

$\eta=\arcsin \left(\sqrt{\cos ^{2}(\xi)+\cos ^{2}(\zeta)}\right), \quad \eta \in\left[0, \frac{\pi}{2}\right]$

Since angles $\xi, \eta$ and $\zeta$ are linked by (6), they are not three independent processes.

\subsection{Statistical modelling of the virtual wall normal vector angles in the case of low roughness}

Let us assume that the virtual wall normal vector angles $\xi$ and $\zeta$ are independent random variables as has been confirmed with the $(\zeta, \xi)$ scatter plots shown in Fig. 4 . The joint probability density function $P_{\xi \zeta}(\theta, \varphi)$ of angles $\xi$ and $\zeta$ can then be written as:

$P_{\xi \zeta}(\theta, \varphi)=P_{\xi}(\theta) P_{\zeta}(\varphi) \quad(\theta, \varphi) \in[0, \pi]^{2}$

The isotropic wall roughness is characterized by equal PDFs of angles $\xi$ and $\zeta$ :

$P_{\xi}(\theta)=P_{\zeta}(\theta), \quad \theta \in[0, \pi]$

with mean values $\bar{\xi}=\bar{\zeta}=\pi / 2$ and standard deviations $\Delta \xi=\Delta \zeta$ of angles $\xi$ and $\zeta$, respectively.

Angles $\xi$ and $\zeta$ may be written in the following form:

$\xi=\frac{\pi}{2}+\xi^{\prime}, \quad \zeta=\frac{\pi}{2}+\zeta^{\prime}$

Further, the case of low roughness is studied which means that the effective values of the vector angles satisfy the relations:

$\left|\xi^{\prime}\right| \ll \frac{\pi}{2}$ and $\left|\zeta^{\prime}\right| \ll \frac{\pi}{2}$ 
Table 1

Virtual wall normal vector angle statistical characteristics (in degrees). The virtual rough walls examined are characterized by the ratio of RMS height to correlation length scale: $h / c_{\mathrm{L}}$. The mean value of the angle $\eta$ is $\bar{\eta}$ and the standard deviations of angles $\xi, \eta$ and $\zeta$ are $\Delta \xi, \Delta \eta$ and $\Delta \zeta$ respectively; the subscript NS stands for numerical simulation and SM stands for the statistical model (16) and (17).

\begin{tabular}{llllllll}
\hline$h / c_{\mathrm{L}}$ & $\Delta \xi_{\mathrm{NS}}$ & $\Delta \zeta_{\mathrm{NS}}$ & $\frac{\sqrt{2} \cdot 180}{\pi} h / c_{\mathrm{L}}$ & $\bar{\eta}_{\mathrm{NS}}$ & $\Delta \eta_{\mathrm{NS}}$ & $\bar{\eta}_{\mathrm{SM}}$ & $\Delta \eta_{\mathrm{SM}}$ \\
\hline 0.031 & 2.42 & 2.45 & 2.51 & 3.05 & 1.60 & 3.03 & 1.58 \\
0.037 & 2.98 & 3.02 & 3.00 & 3.75 & 1.99 & 3.73 & 1.95 \\
0.044 & 3.54 & 3.60 & 3.56 & 4.49 & 2.33 & 4.44 & 2.32 \\
0.050 & 3.97 & 4.00 & 4.05 & 5.03 & 2.58 & 4.97 & 2.60 \\
0.056 & 4.55 & 4.53 & 4.54 & 5.72 & 2.96 & 5.70 & 2.98 \\
0.063 & 5.08 & 5.05 & 5.10 & 6.39 & 3.28 & 6.36 & 3.33 \\
\hline
\end{tabular}

so the corresponding standard deviations of the angle PDFs satisfy:

$\Delta \xi \ll \frac{\pi}{2}$ and $\Delta \zeta \ll \frac{\pi}{2}$

For low roughness angles (in radians), (5) simplifies to,

$\Delta \xi=\Delta \zeta=\sqrt{2} \frac{h}{c_{\mathrm{L}}}$

since $s_{x} \approx \xi^{\prime}$ and $s_{z} \approx \zeta^{\prime}$. It can be seen from Fig. 5 that the low roughness approximation (12) is valid up to approximately 0.15 rad (around $8^{\circ}$ ).

\subsection{Modelled distribution of angle $\eta$ and validation from deterministic simulation}

Using (6), (9) and (10), angle $\eta$ may be written in the frame of the low roughness approximation as,

$\eta=\sqrt{\xi^{\prime 2}+\zeta^{\prime 2}}$

Using (13), the probability density function $P_{\eta}(\theta)$ of angle $\eta$ is obtained by integration of the bi-Gaussian joint probability density function $P_{\xi \zeta}$ on a circle of radius $\eta$ and, for an isotropic rough wall:

$P_{\eta}(\theta)=\frac{\theta}{\Delta \xi^{2}} \exp \left(-\frac{\theta^{2}}{2 \Delta \xi^{2}}\right)$

with, by definition:

$\int_{0}^{\pi / 2} \frac{\theta}{\Delta \xi^{2}} \exp \left(-\frac{\theta^{2}}{2 \Delta \xi^{2}}\right) \mathrm{d} \theta=1$

The mean value and variance of the probability density function of angle $\eta$, defined with (14) are:

$\bar{\eta}=\sqrt{\frac{\pi}{2}} \Delta \xi$

$\Delta \eta^{2}=\left[2-\frac{\pi}{2}\right] \Delta \xi^{2}$

In Table 1 , the mean values and standard deviations of angle $\eta$ obtained from the statistical model (16) and (17) are compared with the angle $\eta$ of the generated virtual surface, for different ratios of RMS height $h$ to correlation length scale $c_{\mathrm{L}}$. The agreement between these compared values is very good. The standard deviations $\Delta \xi$ and $\Delta \zeta$ obtained from the numerical simulation are almost identical as expected for an isotropic rough wall.

\section{Numerical simulation of particle 3D elastic bouncing on a rough wall and statistical analysis}

\subsection{Description of a numerical simulation}

The numerical simulation is realized as follows. An isotropic virtual wall with RMS height $h$ and correlation length scale $c_{\mathrm{L}}$ is generated according to the procedure outlined in Section 2.1. These wall generation parameters lead to virtual normal vector angle deviations $\Delta \xi=\Delta \zeta$ given by (12). In this section virtual walls with virtual normal vector angle deviations $\Delta \xi=\Delta \zeta=2.5^{\circ}$ and $\Delta \xi=\Delta \zeta=5^{\circ}$ are examined.

Starting particle centre coordinates $x$ and $z$ are sampled from the uniform distribution, while the starting $y$ position is the same for all particles and slightly higher than the highest asperity in the simulated domain. The point of impact of the particle on the virtual surface is found and a unit normal vector $\mathbf{n}$ is calculated. Projections of the incident velocity vector $\mathbf{U}_{\mathrm{p}}^{-}$are calculated using transformation matrices in a local coordinate system $\left(x^{\prime}, y^{\prime}, z^{\prime}\right)$, where the $y^{\prime}$ axis is along the virtual wall normal vector $\mathbf{n}$ (particle incident properties are denoted with superscript - and particle rebound properties with superscript + ). Fully elastic impacts are calculated $\left(u_{\mathrm{p}}^{\prime+}=u_{\mathrm{p}}^{\prime-}, v_{\mathrm{p}}^{\prime+}=-v_{\mathrm{p}}^{\prime-}, w_{\mathrm{p}}^{\prime+}=w_{\mathrm{p}}^{\prime-}\right)$ and the velocity components obtained are written back in the general coordinate system $(x, y, z)$. After rebound, the particle is tracked and further impacts with the wall are calculated, if they exist. The particle is tracked until it overshoots the highest asperity in the domain.

10,000 particle trajectories are simulated per simulation, which is sufficient to obtain converged statistics. In the numerical simulation concerning the incident particle velocity, the velocity projection $u_{\mathrm{p}}^{-}$is specified as input data in addition to the angles $\alpha_{\mathrm{p}}^{-}$and $\beta_{\mathrm{p}}^{-}$, as shown in Fig. 6.

\subsection{Statistical analysis of $3 D$ particle rebound from isotropic rough} wall

PDFs of the first virtual normal vector angles $\xi$ and $\zeta$ seen by particles with incident angles $\alpha_{\mathrm{p}}^{-}$and $\beta_{\mathrm{p}}^{-}$, and different virtual walls characterized with virtual normal vector standard deviations $\Delta \xi=\Delta \zeta$, are shown in Fig. 7 and Fig. 8, respectively.

It can be seen from Fig. 7 that at large incident angles $\alpha_{\mathrm{p}}^{-}$ $\left(\left|\alpha_{\mathrm{p}}^{-}\right| \gg \Delta \xi\right.$ and $\left.\Delta \zeta\right)$, the distribution of the first angle $\xi$ seen by incident particles is Gaussian with a mean value equal to $\pi / 2$ and a standard deviation equal to the value calculated from (12). In contrast, as the particle incident angle $\left|\alpha_{\mathrm{p}}^{-}\right|$decreases, the PDF of the first angle $\xi$ seen by incident particles becomes asymmetric with a mean value shifted towards higher values. Both of these effects are enhanced with increase in the virtual wall normal vector standard deviation $\Delta \xi=\Delta \zeta$ from $2.5^{\circ}$ to $5^{\circ}$. These effects may be due the so-called shadow effect as pointed out by Sommerfeld and Huber (1999). For low particle incident angles $\left|\alpha_{\mathrm{p}}^{-}\right|$, the PDFs look very slightly sensitive to the particle incident angle $\beta_{\mathrm{p}}^{-}$.

Fig. 8 shows that the PDF of the first angle $\zeta$ seen by incident particles follows the same trend as the angle $\xi$ with a mean value equal to $\pi / 2$ and a standard deviation equal to that of $\xi$. Also, the PDF becomes dependent on the particle incidence angles $\alpha_{\mathrm{p}}^{-}$and $\beta_{\mathrm{p}}^{-}$with decreasing values of $\left|\alpha_{\mathrm{p}}^{-}\right|$. This effect is more pronounced for high wall roughness values. Therefore, the effect of incident angle $\beta_{\mathrm{p}}^{-}$for small incident angle amplitudes $\alpha_{\mathrm{p}}^{-}$is larger on the PDF of angle $\zeta$ than on the PDF of angle $\xi$.

Figs. 9 and 10 show PDFs of particle bouncing angles $\alpha_{\mathrm{p}}^{+}$and $\beta_{\mathrm{p}}^{+}$computed from deterministic simulations, according to definitions shown in Fig. 6.

Fig. 9 shows the PDFs of angles $\alpha_{\mathrm{p}}^{+}$obtained from deterministic simulations for different incident particle angles $\alpha_{\mathrm{p}}^{-}$and $\beta_{\mathrm{p}}^{-}$. At large particle incident angles $\alpha_{\mathrm{p}}^{-}\left(\left|\alpha_{\mathrm{p}}^{-}\right| \gg \Delta \xi\right.$ and $\left.\Delta \zeta\right)$, the distribution of the rebound angle $\alpha_{\mathrm{p}}^{+}$is nearly Gaussian with a mean value equal to the absolute value of the particle incident angle $\left|\alpha_{\mathrm{p}}^{-}\right|$ and a standard deviation approximately equal to that of the virtual wall normal vector $\Delta \xi=\Delta \zeta$. When the particle incident angle $\left|\alpha_{\mathrm{p}}^{-}\right|$decreases, a strong shift towards small values is observed and the PDF is no longer Gaussian. This behaviour is consistent with the 2D shadow effect analyzed by Sommerfeld and Huber (1999). 

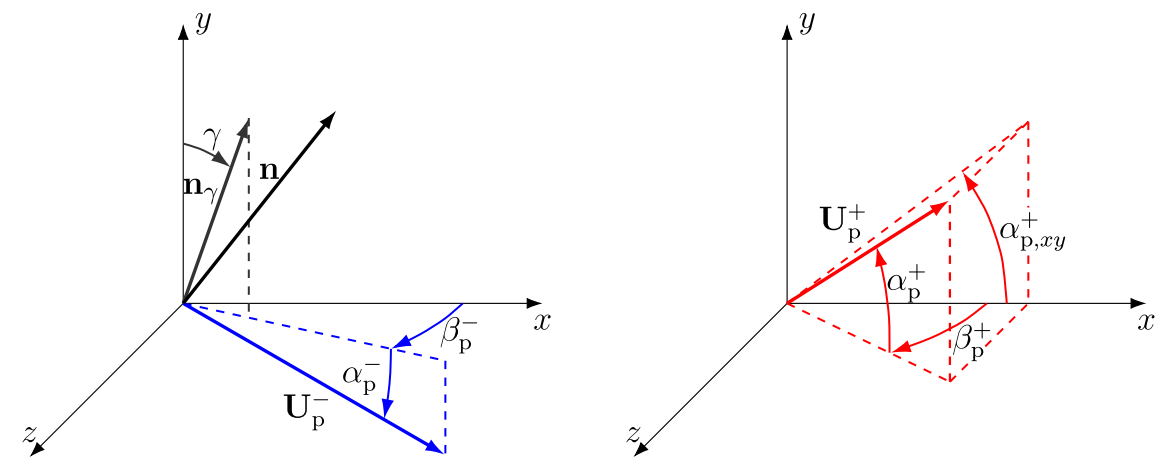

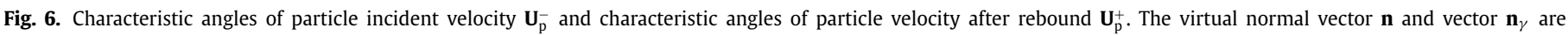
defined in (4) and (21), respectively, and angle $\gamma$ satisfies (23).
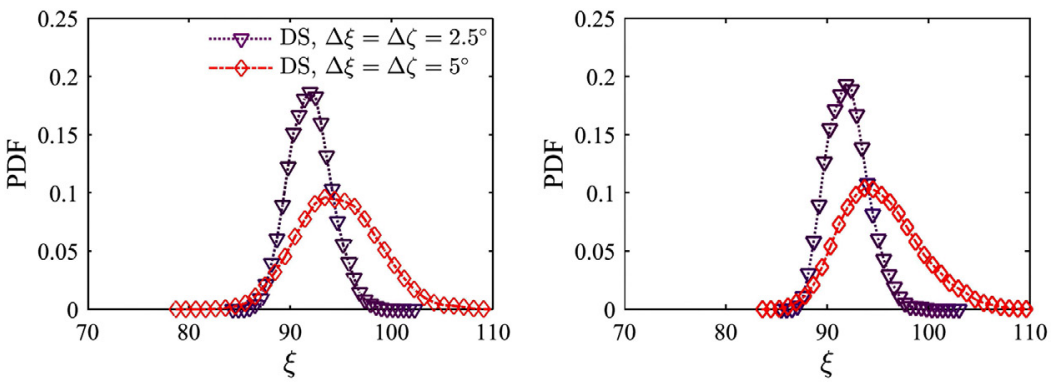

(a) $\alpha_{\mathrm{p}}^{-}=-2.5^{\circ}, \beta_{\mathrm{p}}^{-}=-30^{\circ}$

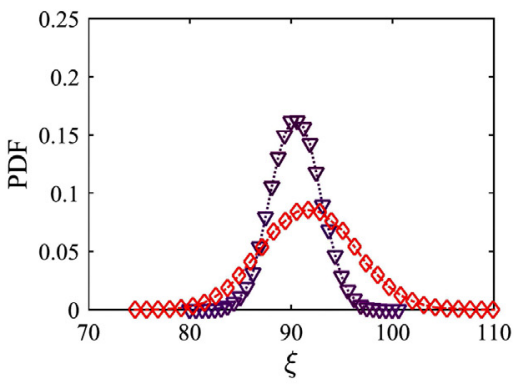

(b) $\alpha_{\mathrm{p}}^{-}=-2.5^{\circ}, \beta_{\mathrm{p}}^{-}=15^{\circ}$

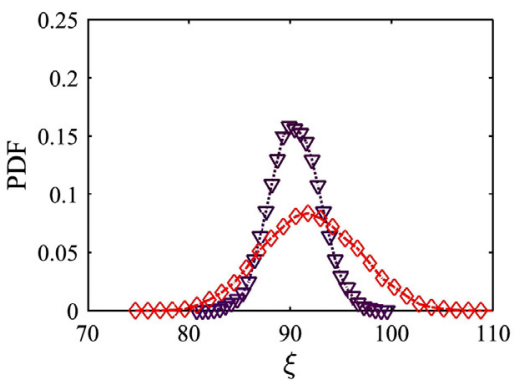

(c) $\alpha_{\mathrm{p}}^{-}=-12.5^{\circ}, \beta_{\mathrm{p}}^{-}=-30^{\circ}$

(d) $\alpha_{\mathrm{p}}^{-}=-12.5^{\circ}, \beta_{\mathrm{p}}^{-}=15^{\circ}$
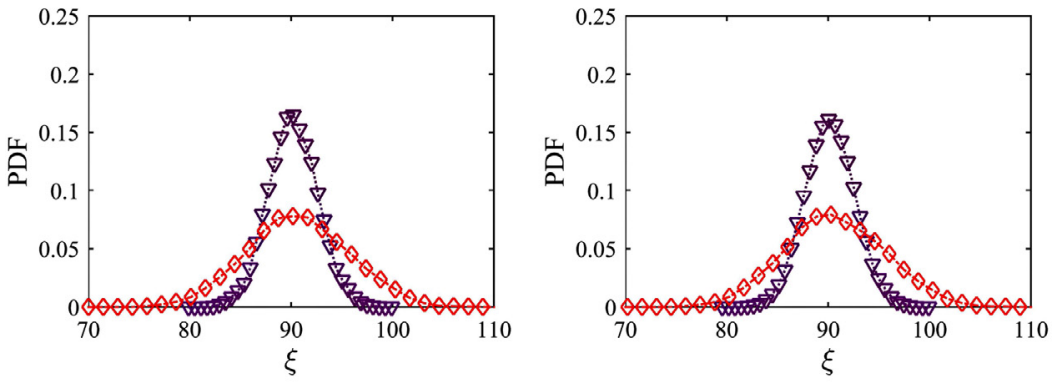

(e) $\alpha_{\mathrm{p}}^{-}=-32.5^{\circ}, \beta_{\mathrm{p}}^{-}=-30^{\circ}$

(f) $\alpha_{\mathrm{p}}^{-}=-32.5^{\circ}, \beta_{\mathrm{p}}^{-}=15^{\circ}$

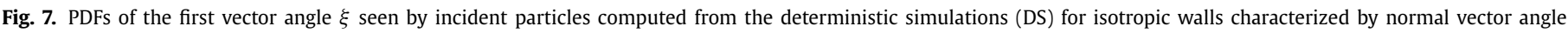
standard deviations $\Delta \xi=\Delta \zeta$, for different particle incident angles $\alpha_{\mathrm{p}}^{-}$and $\beta_{\mathrm{p}}^{-}$.

These PDFs of rebound angle $\alpha_{\mathrm{p}}^{+}$from deterministic simulation are then compared with the results of the $2 \mathrm{D}$ multiple particle-wall collision model of Konan et al. (2009) applied in the incident particle plane. The agreement between these two distributions is very good, which suggests that the 2D model of Konan et al. (2009) can be applied to calculate the final rebound angle $\alpha_{\mathrm{p}}^{+}$in the case of 3D particle rebound from a wall.
However, the 2D approach of Konan et al. (2009) cannot predict the transverse deviation bouncing angle $\beta_{\mathrm{p}}^{+}-\beta_{\mathrm{p}}^{-}$. The PDFs of this angle are shown in Fig. 10, for cases of the first and final particle rebound, for different incident angles $\alpha_{\mathrm{p}}^{-}$and $\beta_{\mathrm{p}}^{-}$and two virtual wall roughness values. These PDFs agree very well for the case of the first and final rebounds, which leads to the conclusion that the transverse angle distribution is not influenced by multiple 

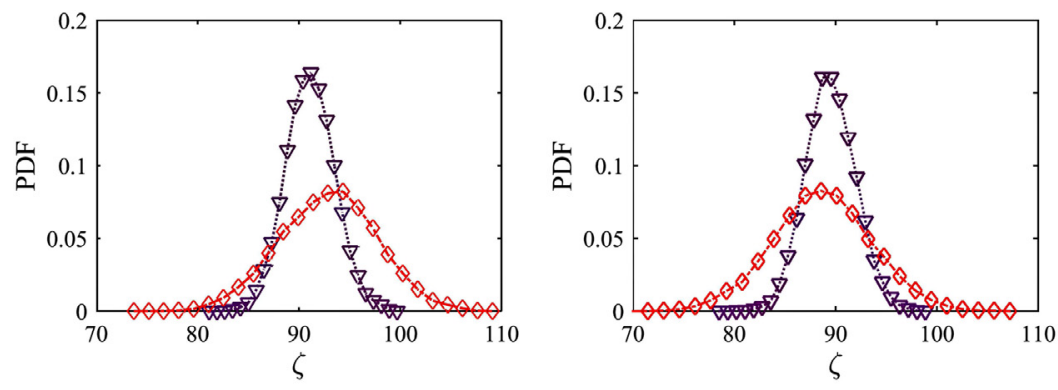

(a) $\alpha_{\mathrm{p}}^{-}=-2.5^{\circ}, \beta_{\mathrm{p}}^{-}=-30^{\circ}$

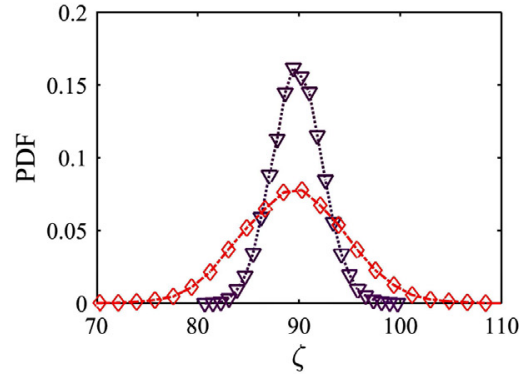

(b) $\alpha_{\mathrm{p}}^{-}=-2.5^{\circ}, \beta_{\mathrm{p}}^{-}=15^{\circ}$

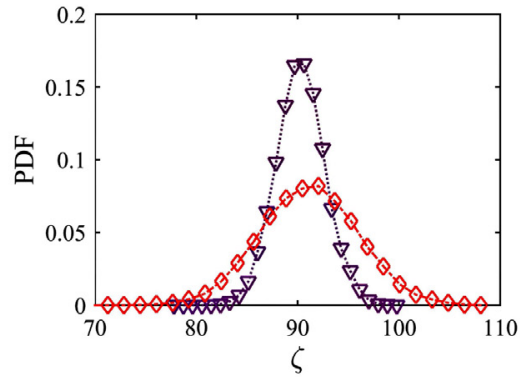

(c) $\alpha_{\mathrm{p}}^{-}=-12.5^{\circ}, \beta_{\mathrm{p}}^{-}=-30^{\circ}$

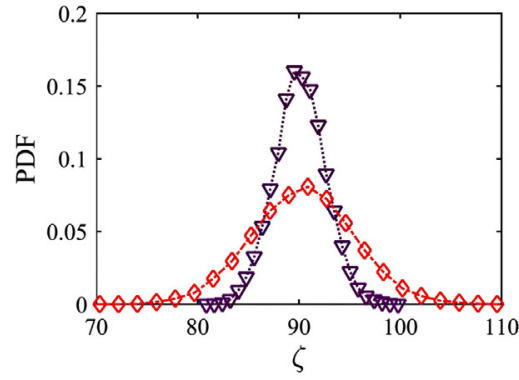

(d) $\alpha_{\mathrm{p}}^{-}=-12.5^{\circ}, \beta_{\mathrm{p}}^{-}=15^{\circ}$

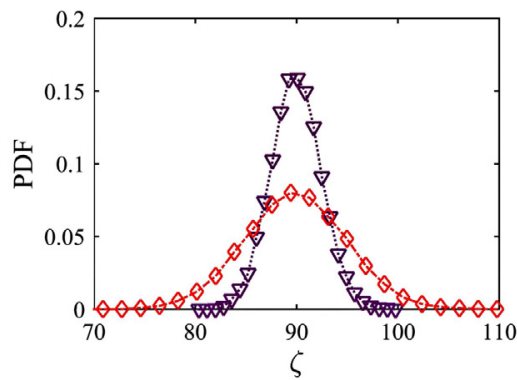

(e) $\alpha_{\mathrm{p}}^{-}=-32.5^{\circ}, \beta_{\mathrm{p}}^{-}=-30^{\circ}$

(f) $\alpha_{\mathrm{p}}^{-}=-32.5^{\circ}, \beta_{\mathrm{p}}^{-}=15^{\circ}$

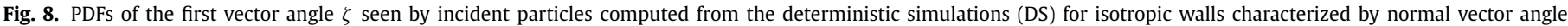
standard deviations $\Delta \xi=\Delta \zeta$, for different particle incident angles $\alpha_{\mathrm{p}}^{-}$and $\beta_{\mathrm{p}}^{-}$. Legend is the same as in Fig. 7 .

particle-wall collisions. It can be seen that the transverse deviation bouncing angle $\beta_{\mathrm{p}}^{+}-\beta_{\mathrm{p}}^{-}$obeys a Gaussian distribution with a standard deviation that increases with increasing incident angle amplitude $\left|\alpha_{\mathrm{p}}^{-}\right|$. It is also observed that this standard deviation increases with increasing virtual normal vector angle standard deviations $\Delta \xi=\Delta \zeta$. The PDFs of the transverse deviation bouncing angle $\beta_{\mathrm{p}}^{+}-\beta_{\mathrm{p}}^{-}$are independent of the incident angle $\beta_{\mathrm{p}}^{-}$which is to be expected, since the virtual walls are isotropic.

\section{Statistical modelling of the 3D rough wall-particle collisions}

4.1. Modelling the first virtual wall normal vector seen by particles for large incident angles

As shown in Figs. 7 and 8, for large particle incident angles $\left|\alpha_{\mathrm{p}}^{-}\right|$, with respect to the angle standard deviations, $\left|\alpha_{\mathrm{p}}^{-}\right| \gg \Delta \xi$ and $\Delta \zeta$, and low wall roughness (11), the first angles $\xi$ and $\zeta$ seen by particles have Gaussian distributions, with zero mean and standard deviation equal to the virtual wall normal vector standard deviations $\Delta \xi=\Delta \zeta$. Compared with this case, for particles with low incident angles $\left|\alpha_{\mathrm{p}}^{-}\right|$, the PDFs of angles $\xi$ and $\zeta$ change since there is a conditioning effect by the incident particle angle $\alpha_{\mathrm{p}}^{-}$.
In this section, statistical modelling is developed for large incident angles, so that we can neglect the shadow effect on the PDF of the wall normal vector angle seen by particles.

According to Fig. 6, the incident particle velocity $\mathbf{U}_{\mathrm{p}}^{-}$can be written,

$\mathbf{U}_{\mathrm{p}}^{-}=\left|\mathbf{U}_{\mathrm{p}}^{-}\right| \cos \left(\alpha_{\mathrm{p}}^{-}\right) \mathbf{t}_{\mathrm{p}}^{-}+\left|\mathbf{U}_{\mathrm{p}}^{-}\right| \sin \left(\alpha_{\mathrm{p}}^{-}\right) \mathbf{j}, \quad \alpha_{\mathrm{p}}^{-} \in\left[-\frac{\pi}{2}, 0\right]$

where $\mathbf{t}_{\mathrm{p}}^{-}$is the unit vector collinear with the projection of the incident particle velocity $\mathbf{U}_{\mathbf{p}}^{-}$on the horizontal plane,

$\mathbf{t}_{\mathrm{p}}^{-}=\cos \left(\beta_{\mathrm{p}}^{-}\right) \mathbf{i}-\sin \left(\beta_{\mathrm{p}}^{-}\right) \mathbf{k}, \quad \beta_{\mathrm{p}}^{-} \in[-\pi, \pi]$

It can be noted that any unit vector $\mathbf{n}$ seen by a given incident particle velocity $\mathbf{U}_{\mathrm{p}}^{-}$must verify,

$\mathbf{U}_{\mathrm{p}}^{-} \cdot \mathbf{n}<0$

This condition is part of the shadow effect leading to realizability conditions for the virtual wall normal unit vector angles.

Let us define the unit vector $\mathbf{n}_{\gamma}$ in the incident plane written in terms of the angle $\gamma$ with the $y$-axis as,

$\mathbf{n}_{\gamma}=-\sin (\gamma) \mathbf{t}_{\mathrm{p}}^{-}+\cos (\gamma) \mathbf{j}, \quad \gamma \in\left[-\frac{\pi}{2}, \frac{\pi}{2}\right]$ 

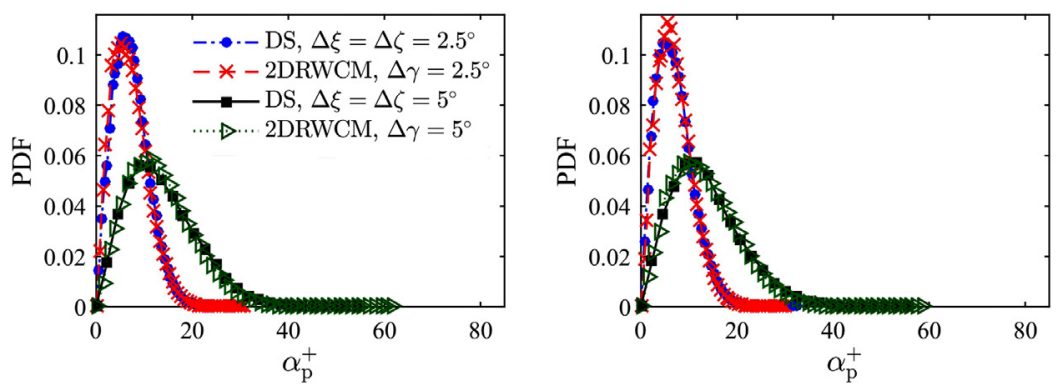

(a) $\alpha_{\mathrm{p}}^{-}=-2.5^{\circ}, \beta_{\mathrm{p}}^{-}=-30^{\circ}$

(b) $\alpha_{\mathrm{p}}^{-}=-2.5^{\circ}, \beta_{\mathrm{p}}^{-}=15^{\circ}$
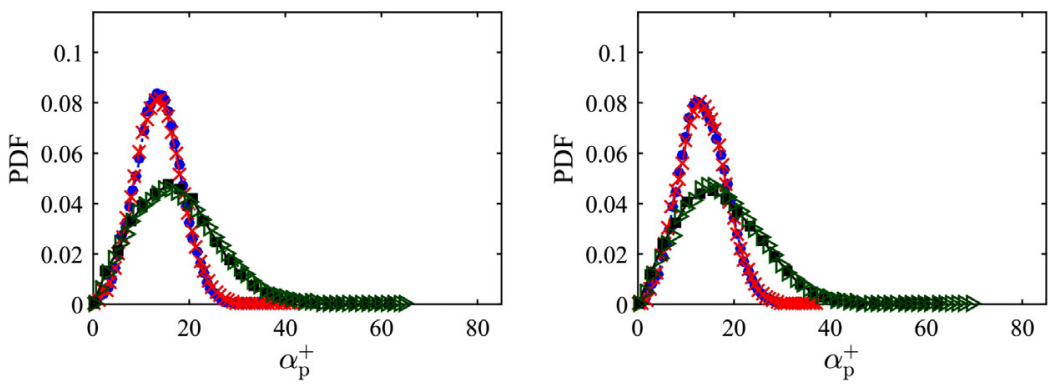

(c) $\alpha_{\mathrm{p}}^{-}=-12.5^{\circ}, \beta_{\mathrm{p}}^{-}=-30^{\circ}$

(d) $\alpha_{\mathrm{p}}^{-}=-12.5^{\circ}, \beta_{\mathrm{p}}^{-}=15^{\circ}$
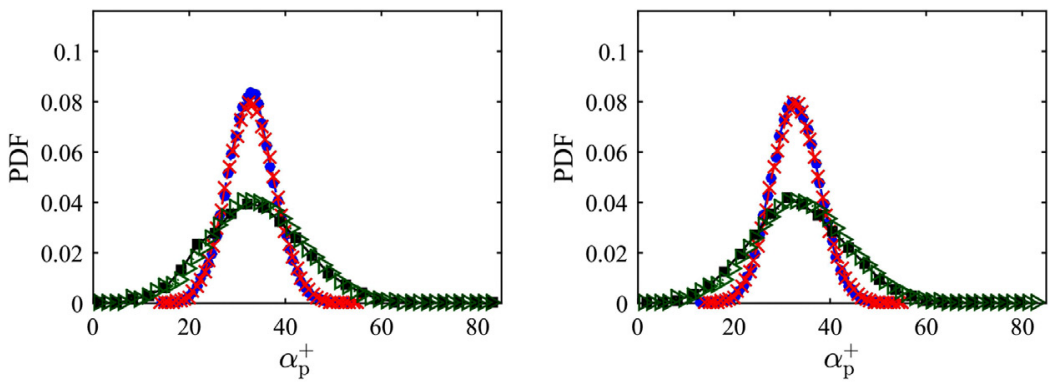

(e) $\alpha_{\mathrm{p}}^{-}=-32.5^{\circ}, \beta_{\mathrm{p}}^{-}=-30^{\circ}$

(f) $\alpha_{\mathrm{p}}^{-}=-32.5^{\circ}, \beta_{\mathrm{p}}^{-}=15^{\circ}$

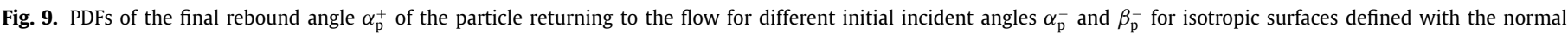

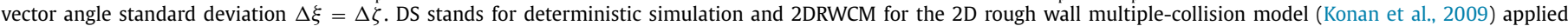
in the particle incident plane for 2D wall normal vector angle standard deviation $\Delta \gamma=\Delta \xi$.

by imposing that the scalar projection of $\mathbf{n}_{\gamma}$ onto the particle incident velocity $\mathbf{U}_{\mathrm{p}}^{-}$is equal to that of the wall normal vector $\mathbf{n}$,

$\mathbf{U}_{\mathrm{p}}^{-} \cdot \mathbf{n}_{\gamma}=\mathbf{U}_{\mathrm{p}}^{-} \cdot \mathbf{n}$

Using (18) and (21), (22) can be written,

$\left|\mathbf{U}_{\mathrm{p}}^{-}\right| \sin \left(\alpha_{\mathrm{p}}^{-}-\gamma\right)=\mathbf{U}_{\mathrm{p}}^{-} \cdot \mathbf{n}$

which is equivalent to the following scalar equation,

$$
\begin{aligned}
\sin \left(\alpha_{\mathrm{p}}^{-}-\gamma\right)= & \cos \left(\beta_{\mathrm{p}}^{-}\right) \cos \left(\alpha_{\mathrm{p}}^{-}\right) \cos (\xi)+\sin \left(\alpha_{\mathrm{p}}^{-}\right) \cos (\eta) \\
& -\sin \left(\beta_{\mathrm{p}}^{-}\right) \cos \left(\alpha_{\mathrm{p}}^{-}\right) \cos (\zeta)
\end{aligned}
$$

The above equation always has a unique solution for $\gamma \in$ $[-\pi / 2, \pi / 2]$. In the frame of the low roughness approximation,

(24) leads to the first-order approximation for $\gamma$,

$\gamma=\cos \left(\beta_{\mathrm{p}}^{-}\right) \xi^{\prime}-\sin \left(\beta_{\mathrm{p}}^{-}\right) \zeta^{\prime}$

For a large particle incident angle $\alpha_{\mathrm{p}}^{-}$and low wall roughness, since angles $\xi^{\prime}$ and $\zeta^{\prime}$ are random independent processes with zero mean values $\overline{\xi^{\prime}}=\overline{\zeta^{\prime}}=0$ and standard deviation $\Delta \xi$ and $\Delta \zeta$, angle $\gamma$ is a random process with zero mean value $\bar{\gamma}=0$ and variance $\Delta \gamma^{2}$ as follows:

$\Delta \gamma^{2}=\cos ^{2}\left(\beta_{\mathrm{p}}^{-}\right) \Delta \xi^{2}+\sin ^{2}\left(\beta_{\mathrm{p}}^{-}\right) \Delta \zeta^{2}$
If angles $\xi^{\prime}$ and $\zeta^{\prime}$ are Gaussian processes, the probability density function $P_{\gamma}(\theta)$ of angle $\gamma$ is Gaussian:

$$
P_{\gamma}(\theta)=\frac{1}{\sqrt{2 \pi \Delta \gamma^{2}}} \exp \left(\frac{-\theta^{2}}{2 \Delta \gamma^{2}}\right), \quad \theta \in\left[-\frac{\pi}{2}, \frac{\pi}{2}\right]
$$

In Fig. 11, the first vector angle $\gamma$ distribution seen by an incident particle in the deterministic simulation is compared with the virtual wall angle distribution obtained from the 2D effective distribution (44) given by Sommerfeld and Huber (1999) calculated in the incident particle plane, with a wall roughness angle standard deviation $\Delta \gamma$ equal to the normal vector angle standard deviation $\Delta \xi=\Delta \zeta$. The agreement between the compared distributions is excellent for any particle incident angle $\alpha_{\mathrm{p}}^{-}$. At large incident angles $\alpha_{\mathrm{p}}^{-}$, the distribution of angle $\gamma$ is nearly Gaussian with $\Delta \gamma=\Delta \xi$, in agreement with the statistical model assumption (27). At low incident amplitude angles $\left|\alpha_{\mathrm{p}}^{-}\right|$, due to the shadow effect, the PDF of angle $\gamma$ shifts to the right. In addition, the PDF of angle $\gamma$ is found to be independent of the transverse incident angle $\beta_{\mathrm{p}}^{-}$.

Let us introduce an additional angle $\gamma^{*}$ such that,

$\gamma^{*}=\sin \left(\beta_{\mathrm{p}}^{-}\right) \xi^{\prime}+\cos \left(\beta_{\mathrm{p}}^{-}\right) \zeta^{\prime}$ 

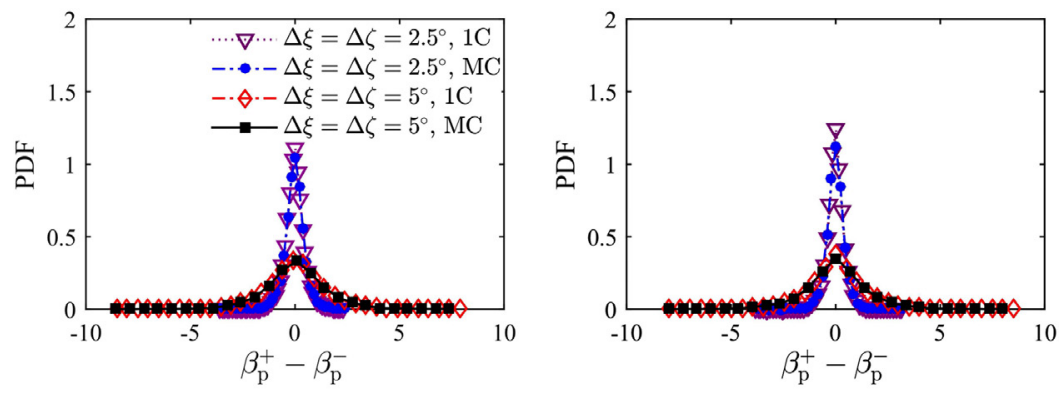

(a) $\alpha_{\mathrm{p}}^{-}=-2.5^{\circ}, \beta_{\mathrm{p}}^{-}=-30^{\circ}$

(b) $\alpha_{\mathrm{p}}^{-}=-2.5^{\circ}, \beta_{\mathrm{p}}^{-}=15^{\circ}$
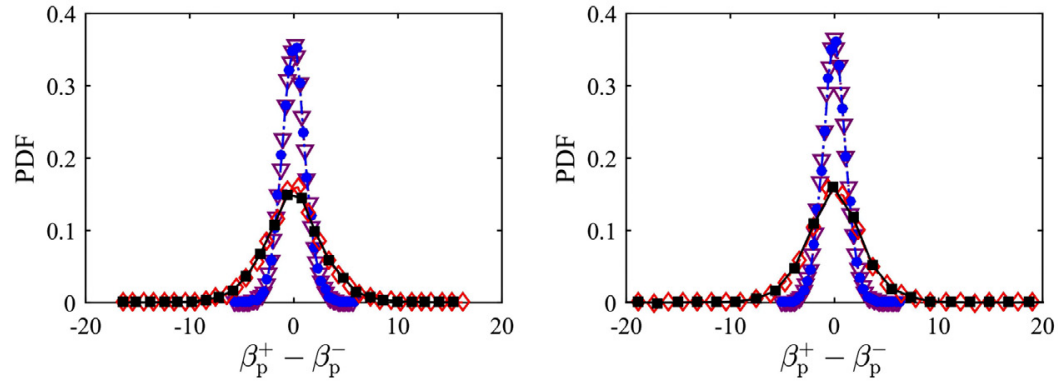

(c) $\alpha_{\mathrm{p}}^{-}=-12.5^{\circ}, \beta_{\mathrm{p}}^{-}=-30^{\circ}$

(d) $\alpha_{\mathrm{p}}^{-}=-12.5^{\circ}, \beta_{\mathrm{p}}^{-}=15^{\circ}$
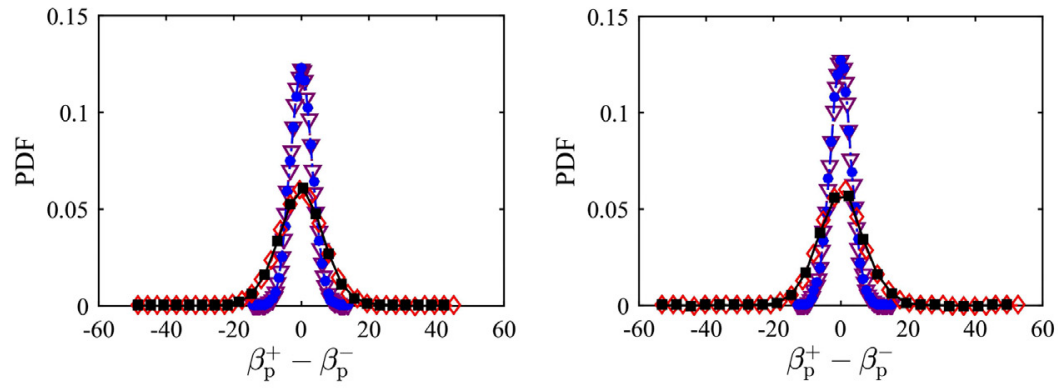

(e) $\alpha_{\mathrm{p}}^{-}=-32.5^{\circ}, \beta_{\mathrm{p}}^{-}=-30^{\circ}$

(f) $\alpha_{\mathrm{p}}^{-}=-32.5^{\circ}, \beta_{\mathrm{p}}^{-}=15^{\circ}$

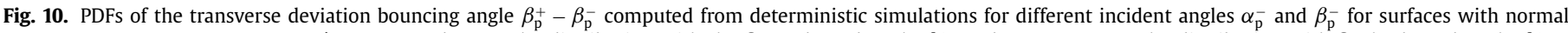
vector angle standard deviations $\Delta \xi=\Delta \zeta$. 1C denotes the distribution with the first rebound angle $\beta_{\mathrm{p}}^{+}$, and MC represents the distribution with final rebound angle $\beta_{\mathrm{p}}^{+}$.

Angle $\gamma^{*}$ is a random process with zero mean value $\overline{\gamma^{*}}=0$ and variance $\Delta \gamma^{* 2}$ expressed as:

$\Delta \gamma^{* 2}=\sin ^{2}\left(\beta_{\mathrm{p}}^{-}\right) \Delta \xi^{2}+\cos ^{2}\left(\beta_{\mathrm{p}}^{-}\right) \Delta \zeta^{2}$

If angles $\xi^{\prime}$ and $\zeta^{\prime}$ are Gaussian processes, probability density function $P_{\gamma^{*}}(\theta)$ of angle $\gamma^{*}$ is Gaussian:

$P_{\gamma^{*}}(\theta)=\frac{1}{\sqrt{2 \pi \Delta \gamma^{* 2}}} \exp \left(\frac{-\theta^{2}}{2 \Delta \gamma^{* 2}}\right), \quad \theta \in\left[-\frac{\pi}{2}, \frac{\pi}{2}\right]$

Fig. 12 shows PDFs of angle $\gamma^{*}$ computed from deterministic simulations using (28) and the model Gaussian distribution given by (30), for the different particle incident angles $\alpha_{\mathrm{p}}^{-}$and $\beta_{\mathrm{p}}^{-}$. The agreement between the two PDFs is excellent. Although angle $\gamma^{*}$ depends on the angles $\xi$ and $\zeta$, as seen in (28), where both angles $\xi$ and $\zeta$ depend on the incident angles $\alpha_{\mathrm{p}}^{-}$and $\beta_{\mathrm{p}}^{-}$, the $\gamma^{*}$ distribution does not depend on the particle incident angles $\alpha_{\mathrm{p}}^{-}$and $\beta_{\mathrm{p}}^{-}$ and, in particular, is not affected by the shadow effect. Hence, by construction, angles $\gamma$ and $\gamma^{*}$ are orthogonal independent Gaussian processes. Finally, for the isotropic rough wall, $\gamma$ and $\gamma^{*}$ are random variables obeying two independent Gaussian distributions with zero means and standard deviations $\Delta \gamma=\Delta \gamma^{*}$, equal to the identical standard deviations $\Delta \xi=\Delta \zeta$ of the virtual wall normal vector angle distributions.
Hence, angles $(\xi, \eta, \zeta)$ of the first virtual rough wall normal vector $\mathbf{n}$ seen by any incident particle can be computed from a pair of random variables $\gamma$ and $\gamma^{*}$ given by independent stochastic processes according to the Gaussian PDF given by (27) and (30). Indeed, using (25) and (28), $\xi$ and $\zeta$ are written,

$\xi=\cos \left(\beta_{\mathrm{p}}^{-}\right) \gamma+\sin \left(\beta_{\mathrm{p}}^{-}\right) \gamma^{*}+\frac{\pi}{2}$

$\zeta=-\sin \left(\beta_{\mathrm{p}}^{-}\right) \gamma+\cos \left(\beta_{\mathrm{p}}^{-}\right) \gamma^{*}+\frac{\pi}{2}$

and, using (13), $\eta$ may be written,

$\eta=\sqrt{\gamma^{2}+\gamma^{* 2}}$

Therefore, for large incident particle velocity angles, no multiple collisions are expected and the first elastic particle-wall collision effect leads to the following expression for the final bouncing particle velocity $\mathbf{U}_{\mathrm{p}}^{+}$:

$\mathbf{U}_{\mathrm{p}}^{+}=\mathbf{U}_{\mathrm{p}}^{-}-2\left[\mathbf{U}_{\mathrm{p}}^{-} \cdot \mathbf{n}\right] \mathbf{n}$

This equation can be written in the following form,

$\mathbf{U}_{\mathrm{p}}^{+}=\mathbf{U}_{\mathrm{p}}^{-}-2\left[\mathbf{U}_{\mathrm{p}}^{-} \cdot \mathbf{n}_{\gamma}\right] \mathbf{n}_{\gamma}-2\left[\mathbf{U}_{\mathrm{p}}^{-} \cdot \mathbf{n}\right]\left[\mathbf{n}-\mathbf{n}_{\gamma}\right]$ 

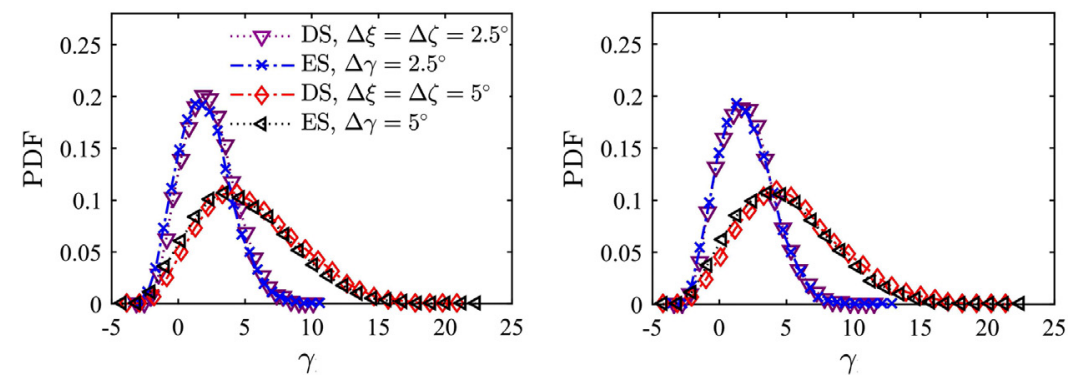

(a) $\alpha_{\mathrm{p}}^{-}=-2.5^{\circ}, \beta_{\mathrm{p}}^{-}=-30^{\circ}$

(b) $\alpha_{\mathrm{p}}^{-}=-2.5^{\circ}, \beta_{\mathrm{p}}^{-}=15^{\circ}$
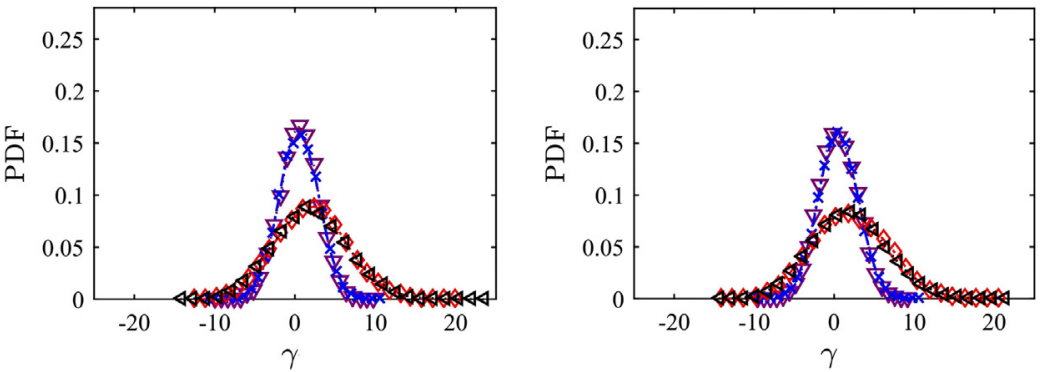

(c) $\alpha_{\mathrm{p}}^{-}=-12.5^{\circ}, \beta_{\mathrm{p}}^{-}=-30^{\circ}$

(d) $\alpha_{\mathrm{p}}^{-}=-12.5^{\circ}, \beta_{\mathrm{p}}^{-}=15^{\circ}$
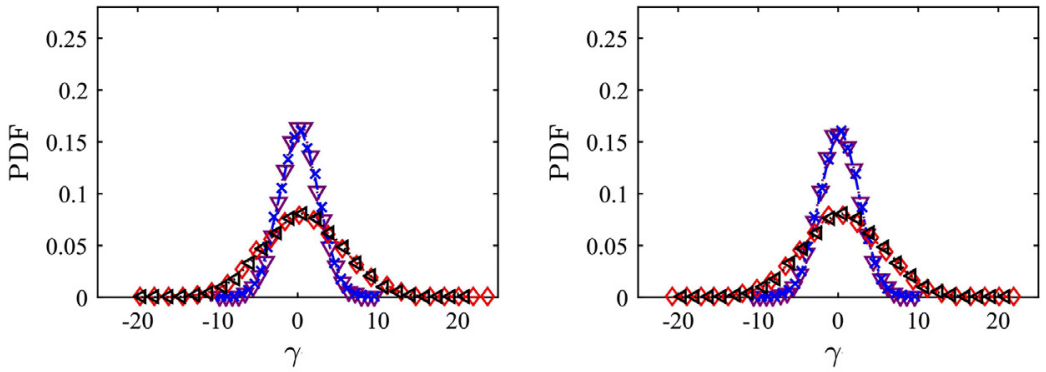

(e) $\alpha_{\mathrm{p}}^{-}=-32.5^{\circ}, \beta_{\mathrm{p}}^{-}=-30^{\circ}$

(f) $\alpha_{\mathrm{p}}^{-}=-32.5^{\circ}, \beta_{\mathrm{p}}^{-}=15^{\circ}$

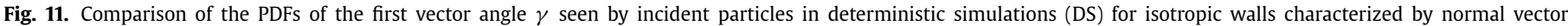

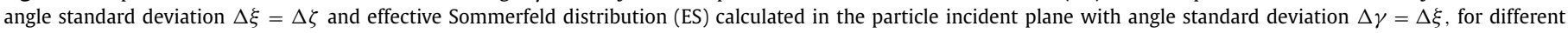
particle incident angles $\alpha_{\mathrm{p}}^{-}$and $\beta_{\mathrm{p}}^{-}$.

where the second term represents the $2 \mathrm{D}$ elastic bouncing of the incident particle on a virtual surface with a normal vector $\mathbf{n}_{\gamma}$ in the incident plane and the third term represents a transverse effect on the particle velocity due to $3 \mathrm{D}$ particle rebound on the isotropic rough wall surface.

4.2. Characterization of transverse bouncing effect for large incident particle angle

Let us define the unit vector normal to particle incident plane:

$\mathbf{s}_{\mathrm{p}}^{-}=\mathbf{t}_{\mathrm{p}}^{-} \wedge \mathbf{j}=\sin \left(\beta_{\mathrm{p}}^{-}\right) \mathbf{i}+\cos \left(\beta_{\mathrm{p}}^{-}\right) \mathbf{k}$

In the frame of the low roughness approximation (11), the transverse virtual wall normal vector component may be written, using (4), (21) and (36), as:

$\mathbf{n}-\mathbf{n}_{\gamma}=-\gamma^{*} \mathbf{s}_{\mathrm{p}}^{-}$

Therefore, according to (35) and (37), the 3D effect on elastic bouncing velocity of particles with large incident particle angle may be written:

$\delta \mathbf{U}_{\mathrm{p}}^{+}=2\left[\mathbf{U}_{\mathrm{p}}^{-} \cdot \mathbf{n}\right] \gamma^{*} \mathbf{s}_{\mathrm{p}}^{-}=w_{\mathrm{p}}^{+} \mathbf{s}_{\mathrm{p}}^{-}$
Eqs. (23) and (38) lead to:

$w_{\mathrm{p}}^{+}=2\left|\mathbf{U}_{\mathrm{p}}^{-}\right| \sin \left(\alpha_{\mathrm{p}}^{-}\right) \gamma^{*}$

Finally, for any given particle incident velocity norm $\left|\mathbf{U}_{\mathrm{p}}^{-}\right|$, velocity $w_{\mathrm{p}}^{+}$is a random variable with zero mean and a standard deviation $\sigma_{\mathrm{p}}^{+}$given by,

$\sigma_{\mathrm{p}}^{+}=2\left|\mathbf{U}_{\mathrm{p}}^{-}\right| \sin \left(\left|\alpha_{\mathrm{p}}^{-}\right|\right) \Delta \gamma^{*}$

By definition, the transverse bouncing angle $\beta_{\mathrm{p}}^{+}-\beta_{\mathrm{p}}^{-}$is written,

$\sin \left(\beta_{\mathrm{p}}^{+}-\beta_{\mathrm{p}}^{-}\right)=-\frac{w_{\mathrm{p}}^{+}}{\mathbf{U}_{\mathrm{p}}^{+} \cos \left(\alpha_{\mathrm{p}}^{+}\right)}$

From (39) and (41), for elastic particle bouncing, it follows that,

$\beta_{\mathrm{p}}^{+}=\beta_{\mathrm{p}}^{-}-2 \tan \left(\alpha_{\mathrm{p}}^{-}\right) \gamma^{*}$

Finally, it is found that angle $\beta_{\mathrm{p}}^{+}-\beta_{\mathrm{p}}^{-}$is a random variable with zero mean and a standard deviation:

$\Delta \beta_{\mathrm{p}}^{+}=2 \tan \left(\left|\alpha_{\mathrm{p}}^{-}\right|\right) \Delta \gamma^{*}$

with $\Delta \gamma^{*}$ given by (29).

Fig. 13 shows the transverse bouncing characteristic parameters, $\sigma_{\mathrm{p}}^{+}$and $\Delta \beta_{\mathrm{p}}^{+}$, due to $3 \mathrm{D}$ elastic rebound on an isotropic rough 

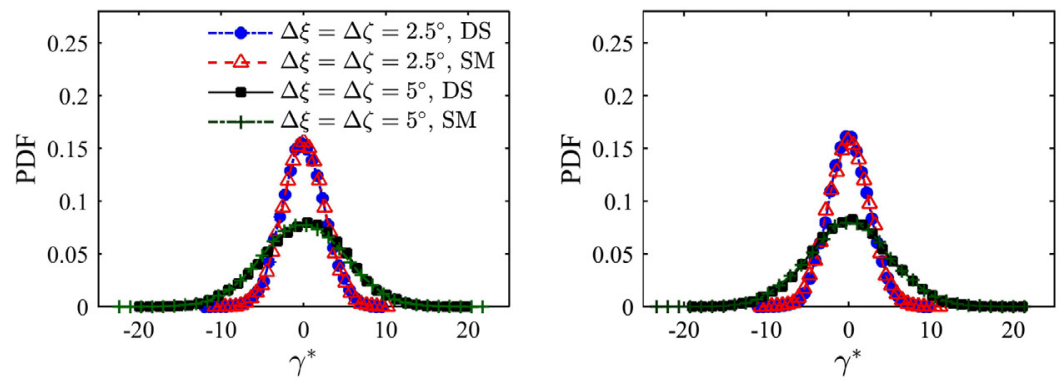

(a) $\alpha_{\mathrm{p}}^{-}=-2.5^{\circ}, \beta_{\mathrm{p}}^{-}=-30^{\circ}$

(b) $\alpha_{\mathrm{p}}^{-}=-2.5^{\circ}, \beta_{\mathrm{p}}^{-}=15^{\circ}$
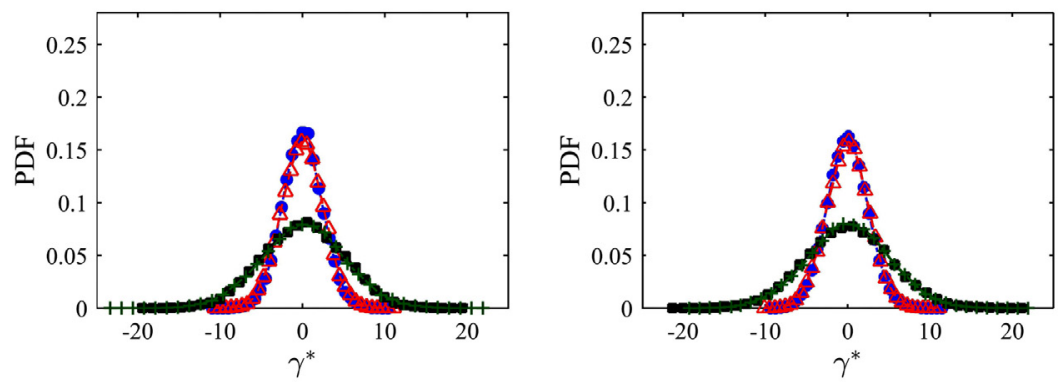

(c) $\alpha_{\mathrm{p}}^{-}=-12.5^{\circ}, \beta_{\mathrm{p}}^{-}=-30^{\circ}$

(d) $\alpha_{\mathrm{p}}^{-}=-12.5^{\circ}, \beta_{\mathrm{p}}^{-}=15^{\circ}$
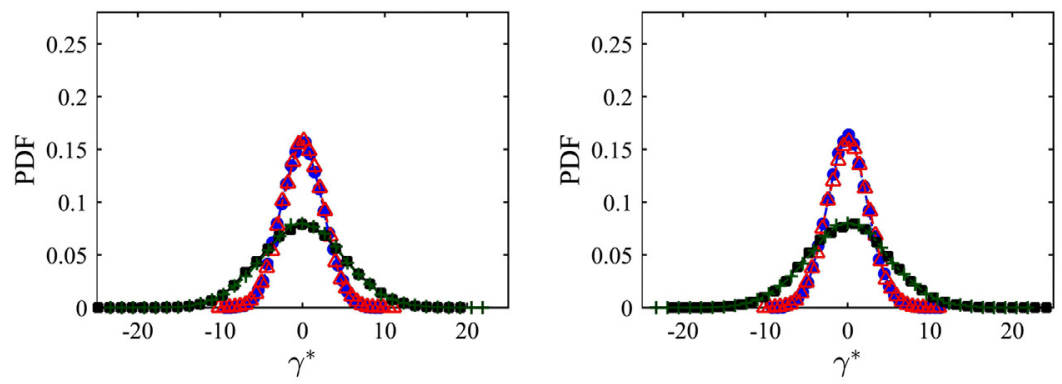

(e) $\alpha_{\mathrm{p}}^{-}=-32.5^{\circ}, \beta_{\mathrm{p}}^{-}=-30^{\circ}$

(f) $\alpha_{\mathrm{p}}^{-}=-32.5^{\circ}, \beta_{\mathrm{p}}^{-}=15^{\circ}$

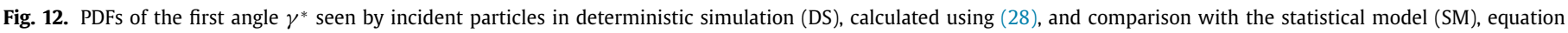
(30), for different particle incident angles $\alpha_{\mathrm{p}}^{-}$and $\beta_{\mathrm{p}}^{-}$.

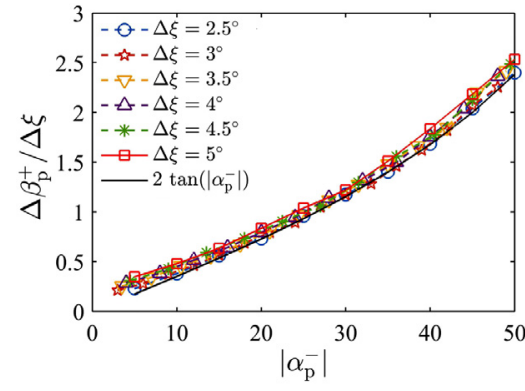

(a)

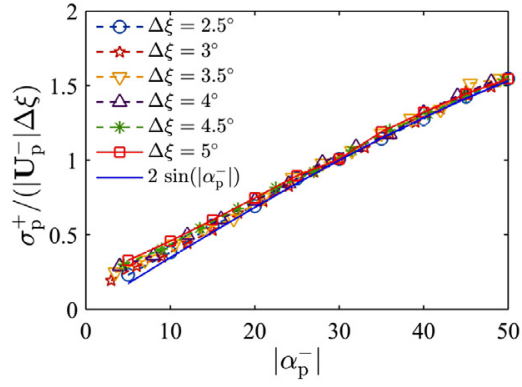

(b)

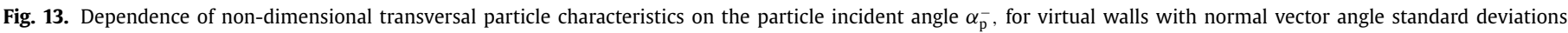

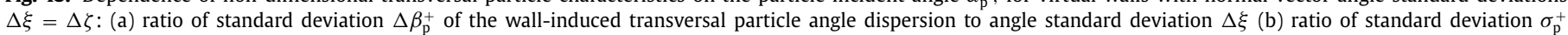
of the wall- induced transversal velocity dispersion to the product of incident velocity norm $\left|\mathbf{U}_{\mathrm{p}}^{-}\right|$and angle standard deviation $\Delta \xi$. 
wall, in terms of the particle incident angle $\alpha_{\mathrm{p}}^{-}$. The agreement between the parameters from deterministic simulations and model predictions, using (40) and (43), is excellent, even for low values of the particle incident angle amplitude $\left|\alpha_{\mathrm{p}}^{-}\right|$.

\subsection{Modelling the first virtual wall normal vector seen by a particle for any incident angle}

For low incident particle velocity angle $\alpha_{\mathrm{p}}^{-}$, of the order of the virtual wall normal vector angle $\gamma$, the statistical approach proposed in Section 4.1 to represent the virtual wall normal vector $\mathbf{n}$ does not ensure that the realizability condition $\mathbf{U}_{\mathrm{p}}^{-} \cdot \mathbf{n}<0$ is satisfied. This realizability condition is part of the shadow effect described by Sommerfeld and Huber (1999) for 2D rebound from a rough wall. They proposed a modified virtual wall normal vector angle probability distribution, conditioned by the incident velocity, which satisfies, by construction, the realizability condition. In the proposed 3D approach, according to (23), the realizability condition may be written as $\gamma>\alpha_{\mathrm{p}}^{-}$and leads to the use of the modified PDF for the $\gamma$ angle alone. Hence, $\gamma^{*}$ is assumed to obey the standard Gaussian distribution given by (30) whereas $\gamma$ is assumed to obey the effective Sommerfeld distribution. Therefore, in practice, angles $\gamma$ and $\gamma^{*}$ are computed from two independent random processes $\left(\gamma, \gamma^{*}\right) \in[-\pi / 2, \pi / 2] \times[-\pi / 2, \pi / 2]$ :

- Angle $\gamma$ obeys the effective Sommerfeld distribution accounting for the shadow effect given by:

if $\gamma>\alpha_{\mathrm{p}}^{-}$:

$P_{e f f}\left(\gamma \mid \alpha_{\mathrm{p}}^{-}\right)=\frac{1}{\sqrt{2 \pi \Delta \gamma^{2}}} \frac{\sin \left(\alpha_{\mathrm{p}}^{-}-\gamma\right)}{\sin \left(\alpha_{\mathrm{p}}^{-}\right)} \exp \left(-\frac{\gamma^{2}}{2 \Delta \gamma^{2}}\right) g\left(\alpha_{\mathrm{p}}^{-}, \gamma\right)$

if $\gamma \leq \alpha_{\mathrm{p}}^{-}$:

$P_{\text {eff }}\left(\gamma \mid \alpha_{\mathrm{p}}^{-}\right)=0$

with

$$
\begin{array}{r}
g\left(\alpha_{\mathrm{p}}^{-}, \Delta \gamma\right)=1 / \int_{\alpha_{\mathrm{p}}^{-}}^{\pi / 2} \frac{1}{\sqrt{2 \pi \Delta \gamma^{2}}} \frac{\sin \left(\alpha_{\mathrm{p}}^{-}-\gamma\right)}{\sin \left(\alpha_{\mathrm{p}}^{-}\right)} . \\
\exp \left(-\frac{\gamma^{2}}{2 \Delta \gamma^{2}}\right) \mathrm{d} \gamma
\end{array}
$$

and standard deviation $\Delta \gamma$ that follows from (26).

- Angle $\gamma^{*}$ obeys a standard Gaussian PDF given by (30).

Finally, the first virtual rough wall normal vector angles are computed from Eqs. (31)-(33).

\subsection{Modelling of multiple particle collisions with the virtual wall}

Sommerfeld and Huber (1999) pointed out that the postrebound condition $\alpha_{\mathrm{p}}^{+}>0$ is needed to allow the particle to return to the main flow. In their applications, they overcame this problem simply by repeating the particle-wall rebound procedure with the generation of a new first virtual wall normal vector angle $\gamma$. Therefore, Konan et al. (2009) showed that "grazing" particles 2D bouncing with a small $\alpha_{\mathrm{p}}^{+}>0$ may suffer several particle-wall collisions before going back to the main flow. Their approach is based on an analytical model of the probability of having only one particlewall collision and on the repetition of the particle-wall rebound procedure in the case of a multiple particle-wall collision effect. The same methodology may be extended directly for 3D particle rebound from rough wall.

The standard deviation $\Delta \beta_{\mathrm{p}}^{+}$remains small, of the order of $\Delta \xi$, as shown in Fig. 13, and decreases for particles with small incident angles that have the highest probability of enduring multiple collisions. Hence, we propose to neglect the 3D deviation effect on the multiple particle-wall collision probability modelling. Therefore, according to Konan et al. (2009), the probability that particles for a given incident angle $\alpha_{\mathrm{p}}^{+}$make only one impact is written as:

$P^{*}\left(n=1 \mid \alpha_{\mathrm{p}}^{+}\right)= \begin{cases}\tanh \left(\psi \frac{\alpha_{\mathrm{p}}^{+}}{\Delta \gamma}\right) & \text { if } \alpha_{\mathrm{p}}^{+} \geq 0 \\ 0 & \text { if } \alpha_{\mathrm{p}}^{+}<0\end{cases}$

with $\psi=1.5$.

Fig. 14 compares the probabilities that particles make only one impact at different particle incident angles $\alpha_{\mathrm{p}}^{-}$and $\beta_{\mathrm{p}}^{-}$in deterministic simulation with the theoretical probability that particles make only one impact (47), with varied coefficient $\psi$. Better agreement with deterministic simulations is obtained for $\psi=2$ than for $\psi=1.5$, probably because the procedure for generating the surface is not identical with that used by Konan et al. (2009). This difference in coefficient $\psi$ does not have a significant effect on the particle rebound statistics and the original value $\psi=1.5$ is retained for this paper.

It follows that the probability that particles make only one impact does not depend on the transverse incident angle $\beta_{\mathrm{p}}^{-}$and transverse rebound angle $\beta_{\mathrm{p}}^{+}$:

$\mathrm{P}^{*}\left(n=1 \mid \alpha_{\mathrm{p}}^{+}, \beta_{\mathrm{p}}^{+}, \beta_{\mathrm{p}}^{-}\right)=\mathrm{P}^{*}\left(n=1 \mid \alpha_{\mathrm{p}}^{+}\right)$

4.5. Stochastic procedure for calculation of 3D rebound of a particle from an isotropic rough wall with low roughness in a Lagrangian framework

In a Lagrangian framework, particles are tracked in the flow and when the centre of any particle reaches half of the particle diameter distance from the boundary surface, the modelling of the effective virtual rough wall is carried out according to the following new stochastic procedure, derived in agreement with the statistical model proposed in Section 4.3 to represent $\gamma$ and $\gamma^{*}$ PDFs.

This procedure can be summarized into five steps:

(1) Angle $\gamma^{*}$ is sampled according to the Gaussian distribution (30)

(2) Angle $\gamma$ is sampled according to the effective Sommerfeld distribution (44)

(3) Angles $\xi, \eta$ and $\zeta$ are calculated with (31)-(33). The virtual wall normal vector $\mathbf{n}$ is defined with (4).

(4) A sliding or non-sliding impact is calculated for a particle colliding with the virtual smooth wall determined with the normal vector $\mathbf{n}$ found in step (3)

(5) Multiple collisions are treated as in Konan et al. (2009).

(5.1) if the rebound angle $\alpha_{\mathrm{p}}^{+} \leq 0$, the procedure is repeated from step (2).

(5.2) if the rebound angle $\alpha_{\mathrm{p}}^{+}>0$, another number $s \in[0,1]$ is sampled according to the uniform distribution.

(5.2.1) if $s \in\left[0, P^{*}\left(n=1 \mid \alpha_{\mathrm{p}}^{+}\right)\right]$, the particle leaves wall region

(5.2.2) if $s \in\left[P^{*}\left(n=1 \mid \alpha_{\mathrm{p}}^{+}\right), 1\right]$, the procedure is repeated from step (2).

4.6. Validation of the modelled virtual wall normal angles at the first particle rebound

Fig. 15 compares the PDFs of virtual wall normal vector angles $\xi, \eta$ and $\zeta$ at the first wall impact from deterministic simulation with the corresponding modelled virtual wall normal vector angles obtained from the procedure outlined in Section 4.5, steps from (1) to (4), since only the first wall impact is modelled. Different incident angles $\alpha_{\mathrm{p}}^{-}$and $\beta_{\mathrm{p}}^{-}$and two isotropic virtual walls are examined. The agreement between the 3D deterministic simulation and 3D stochastic model is very good. 


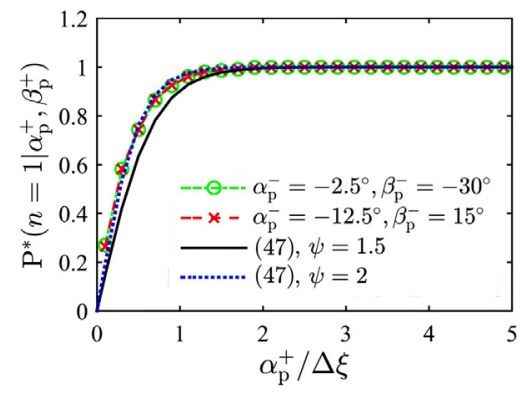

(a) $\Delta \xi=\Delta \zeta=2.5^{\circ}$

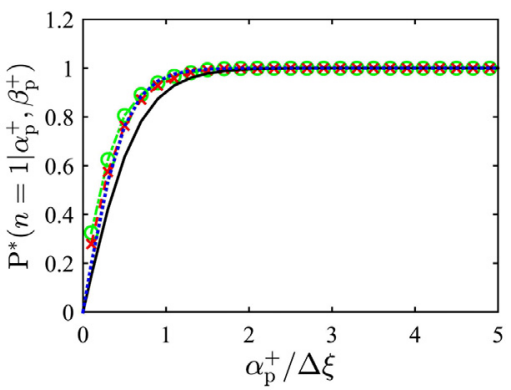

(b) $\Delta \xi=\Delta \zeta=5^{\circ}$

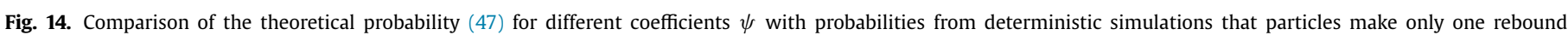
before leaving the wall region, for an isotropic wall with virtual wall normal vector angle standard deviations $\Delta \xi=\Delta \zeta$, for different particle incident angles $\alpha_{\mathrm{p}}^{-}$and $\beta_{\mathrm{p}}^{-}$.

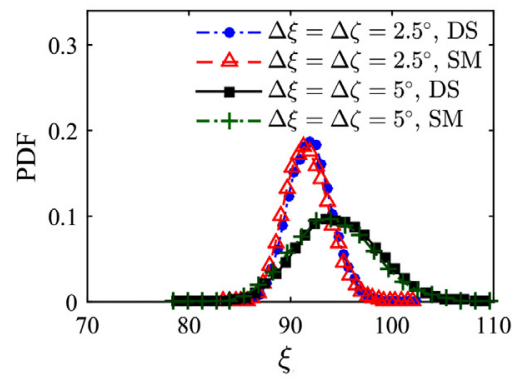

(a) $\alpha_{\mathrm{p}}^{-}=-2.5^{\circ}, \beta_{\mathrm{p}}^{-}=-30^{\circ}$

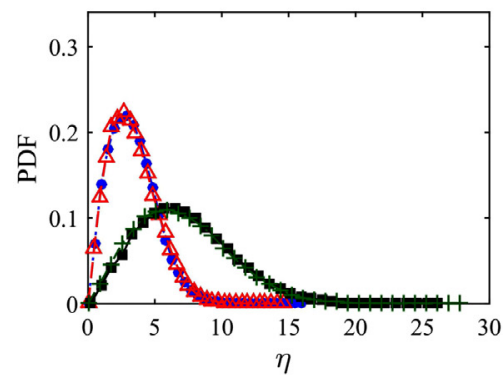

(c) $\alpha_{\mathrm{p}}^{-}=-2.5^{\circ}, \beta_{\mathrm{p}}^{-}=-30^{\circ}$

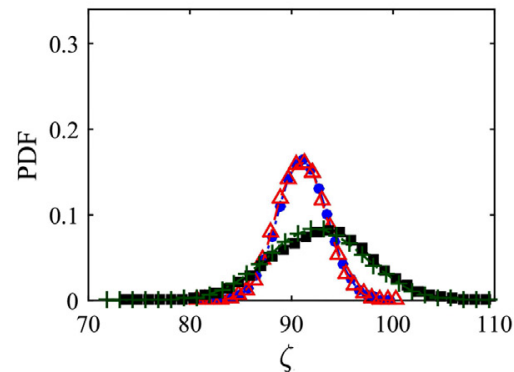

(e) $\alpha_{\mathrm{p}}^{-}=-2.5^{\circ}, \beta_{\mathrm{p}}^{-}=-30^{\circ}$

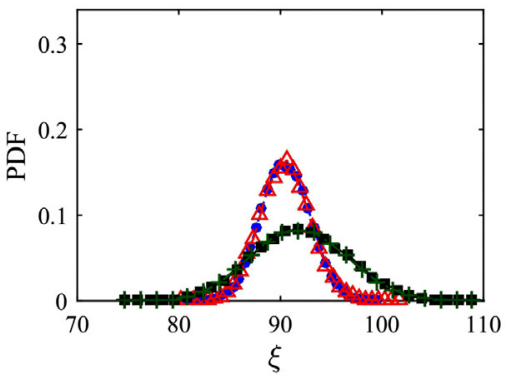

(b) $\alpha_{\mathrm{p}}^{-}=-12.5^{\circ}, \beta_{\mathrm{p}}^{-}=15^{\circ}$

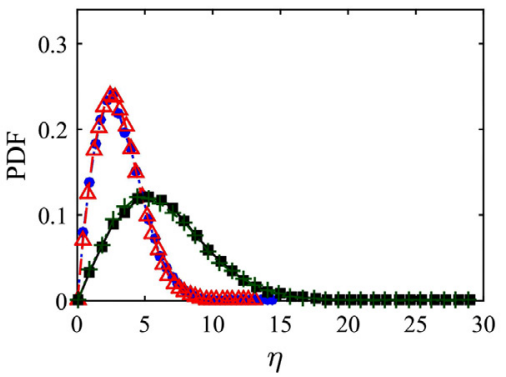

(d) $\alpha_{\mathrm{p}}^{-}=-12.5^{\circ}, \beta_{\mathrm{p}}^{-}=15^{\circ}$

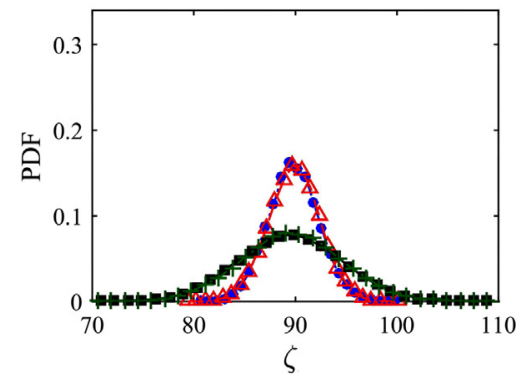

(f) $\alpha_{\mathrm{p}}^{-}=-12.5^{\circ}, \beta_{\mathrm{p}}^{-}=15^{\circ}$

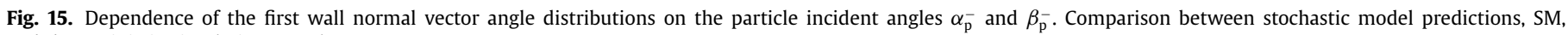
and deterministic simulations results, DM. 

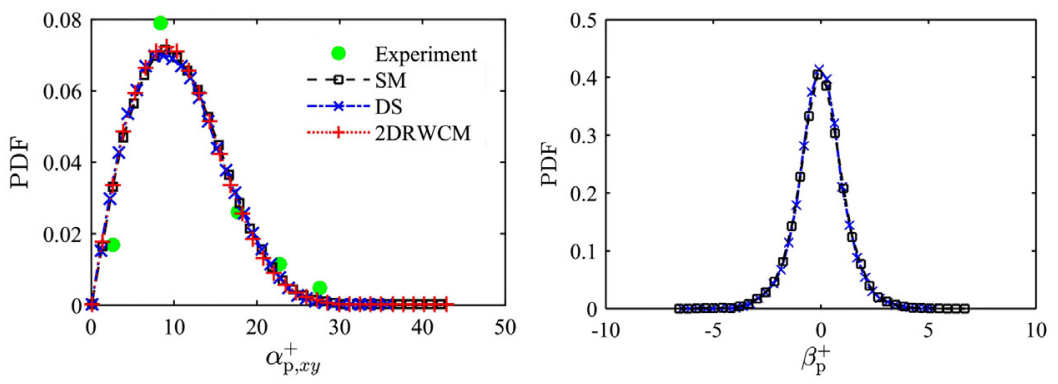

(a) $\alpha_{\mathrm{p}}^{-}=-5^{\circ}$

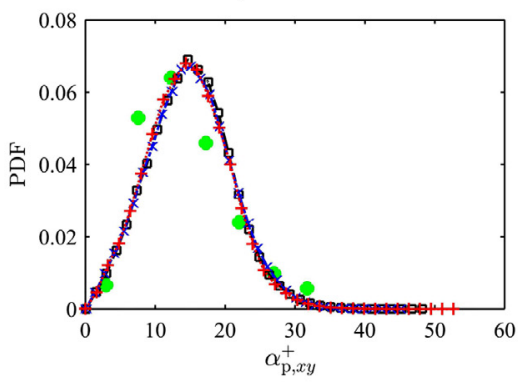

(b) $\alpha_{\mathrm{p}}^{-}=-5^{\circ}, \Delta \beta_{\mathrm{p}}^{+}=1.2^{\circ}$

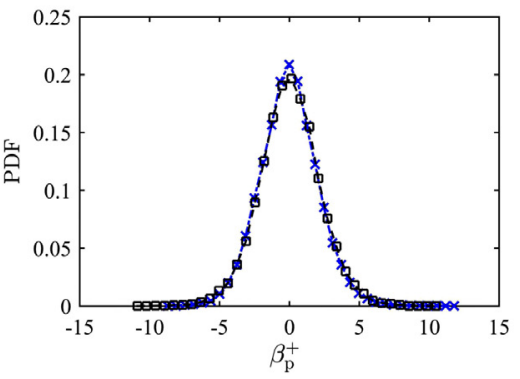

(c) $\alpha_{\mathrm{p}}^{-}=-15^{\circ}$

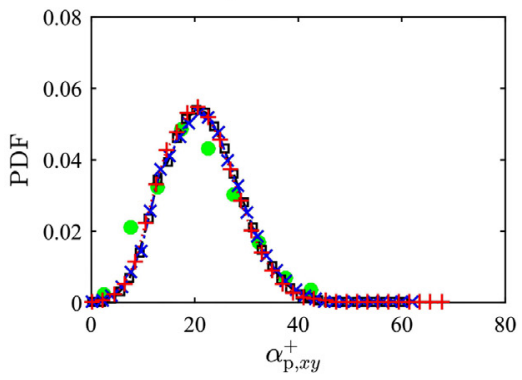

(d) $\alpha_{\mathrm{p}}^{-}=-15^{\circ}, \Delta \beta_{\mathrm{p}}^{+}=2.2^{\circ}$

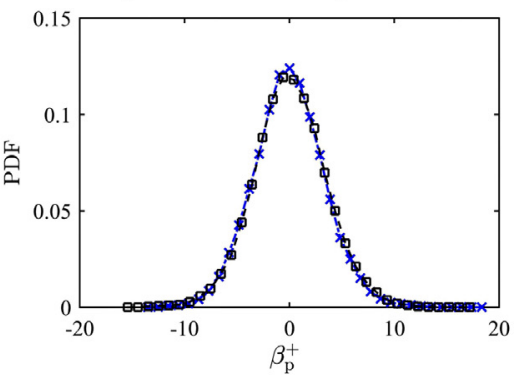

(e) $\alpha_{\mathrm{p}}^{-}=-25^{\circ}$

(f) $\alpha_{\mathrm{p}}^{-}=-25^{\circ}, \Delta \beta_{\mathrm{p}}^{+}=3.5^{\circ}$

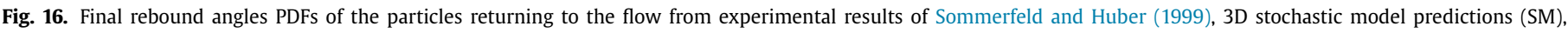

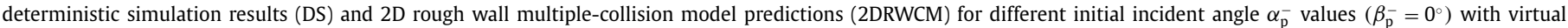
wall normal vector angle standard deviation $\Delta \xi=\Delta \zeta=3.8^{\circ}$.

\section{Applications}

5.1. Comparison of PDFs of particle rebound angles from the experimental measurements and, deterministic and stochastic simulations

In Fig. 16 the derived stochastic model is compared in the $x-y$ plane with the experimental measurements of Sommerfeld and Huber (1999), the 2D rough wall multiple-collision model and the 3D deterministic simulation, whereas in the $y-z$ plane the 3D stochastic model is compared only with the 3D deterministic simulation, since there are no available experimental results in that plane. In order to compare the results of the 3D stochastic model and the 3D deterministic simulation with the available experimental measurements in the $x-y$ plane, angle $\alpha_{\mathrm{p}, x y}^{+}$(Fig. 6) is defined as:

$\alpha_{\mathrm{p}, x y}^{+}=\arctan \left(\frac{\tan \left(\alpha_{p}^{+}\right)}{\cos \left(\beta_{p}^{+}\right)}\right)$

A total of 100,000 particle impacts are simulated with the stochastic method and also with deterministic simulation.

For the experimental configuration of Sommerfeld and $\mathrm{Hu}-$ ber (1999), the wall was made of steel and the particles were made of glass with a diameter $D_{\mathrm{p}}=500 \mu \mathrm{m}$. The wall roughness angle standard deviation was $\Delta \gamma=3.8^{\circ}$ and the horizontal particle mean velocity projection was $u_{\mathrm{p}}^{-}=5.91 \mathrm{~m} / \mathrm{s}$ with root mean square value $u_{\mathrm{RMS}}=1.16 \mathrm{~m} / \mathrm{s}$. Angular velocity was not measured in the experiment, so angular velocity mean and root mean square values are estimated according to the principle outlined in Sommerfeld and Huber (1999) as $\omega_{\mathrm{p} z}=16,336 \mathrm{rad} / \mathrm{s}$ and $\omega_{\mathrm{p} z, \mathrm{RMS}}=$ $5655 \mathrm{rad} / \mathrm{s}$, respectively, as also used by Konan et al. (2009). The flow regime in the experiment was hydraulically smooth.

The coefficient of restitution $e_{\mathrm{w}}$ and the friction coefficient $\mu_{\mathrm{w}}$ depend on the particle incident angle $\alpha_{\mathrm{p}}^{-}$, as defined by Sommerfeld and Huber (1999):

$e_{\mathrm{w}}\left(\alpha_{\mathrm{p}}^{-}\right)= \begin{cases}\frac{e_{\mathrm{h}}-1}{\alpha_{\mathrm{e}}}\left|\alpha_{\mathrm{p}}^{-}\right|+1 & \text { if }\left|\alpha_{\mathrm{p}}^{-}\right| \in\left[0, \alpha_{\mathrm{e}}\right] \\ e_{\mathrm{h}} & \text { if }\left|\alpha_{\mathrm{p}}^{-}\right|>\alpha_{\mathrm{e}}\end{cases}$

and

$\mu_{\mathrm{w}}\left(\alpha_{\mathrm{p}}^{-}\right)= \begin{cases}\frac{\mu_{\mathrm{h}}-\mu_{0}}{\alpha_{\mu}}\left|\alpha_{\mathrm{p}}^{-}\right|+\mu_{0} & \text { if }\left|\alpha_{\mathrm{p}}^{-}\right| \in\left[0, \alpha_{\mathrm{e}}\right] \\ e_{\mathrm{h}} & \text { if }\left|\alpha_{\mathrm{p}}^{-}\right|>\alpha_{\mathrm{e}}\end{cases}$

To match the virtual wall roughness angle standard deviation from the experimental case $\Delta \gamma=3.8^{\circ}$, in deterministic simulations the virtual surface has a ratio of RMS height to correlation 

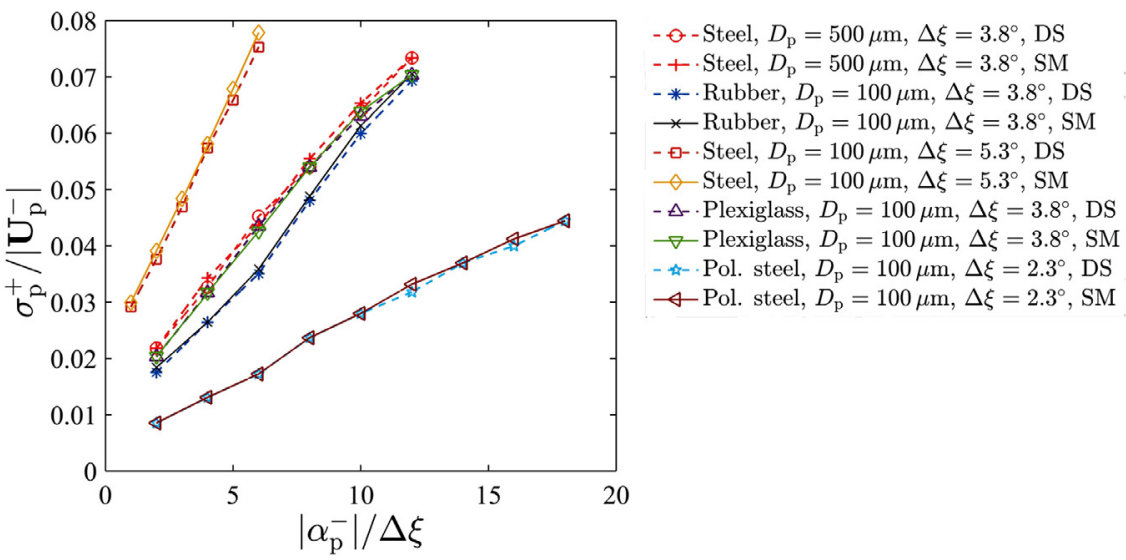

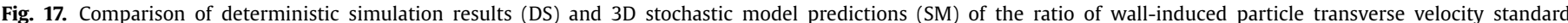

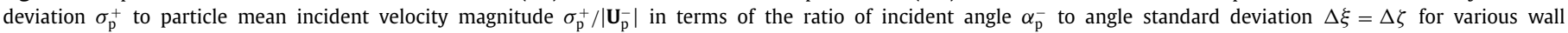
materials and glass particles with diameters $D_{\mathrm{p}}$.

length scale $h / c_{\mathrm{L}}=0.047$ which results in good agreement with the $2 \mathrm{D}$ rough wall multiple-collision model for fully elastic collisions.

Since the exact particle incident angles $\beta_{\mathrm{p}}^{-}$from the experiment are unknown, in the 3D stochastic model and 3D deterministic simulation, the particles have zero incident angle $\beta_{\mathrm{p}}^{-}$. In this way, the rebound of particles in the $y-z$ plane is a direct consequence of the $3 \mathrm{D}$ character of the roughness.

In $3 \mathrm{D}$ procedures, particle collision with a wall is calculated according to Tsuji et al. (1987). A non-sliding collision occurs if the following condition holds:

$v_{\mathrm{p}}^{-}<\frac{-2}{7 \mu_{\mathrm{w}}\left(1+e_{\mathrm{w}}\right)}|\mathbf{U}|$

where the intensity of the velocity $|\mathbf{U}|$ between the particle and the wall at the contact point is:

$|\mathbf{U}|=\sqrt{\left(u_{\mathrm{p}}^{-}+\frac{D_{\mathrm{p}}}{2} \omega_{\mathrm{p} z}^{-}\right)^{2}+\left(w_{\mathrm{p}}^{-}-\frac{D_{\mathrm{p}}}{2} \omega_{\mathrm{p} x}^{-}\right)^{2}}$

The set of equations for the non-sliding collision is:

$$
\begin{aligned}
u_{\mathrm{p}}^{+} & =\frac{5}{7}\left(u_{x}^{-}-\frac{D_{\mathrm{p}}}{5} \omega_{\mathrm{p} z}^{-}\right) \\
v_{\mathrm{p}}^{+} & =-e_{\mathrm{w}} v_{\mathrm{p}}^{-} \\
w_{\mathrm{p}}^{+} & =\frac{5}{7}\left(w_{\mathrm{p}}^{-}+\frac{D_{\mathrm{p}}}{5} \omega_{\mathrm{p} x}^{-}\right) \\
\omega_{\mathrm{p} x}^{+} & =\frac{2 w_{\mathrm{p}}^{-}}{D_{\mathrm{p}}} \\
\omega_{\mathrm{p} y}^{+} & =\omega_{\mathrm{p} y}^{-} \\
\omega_{\mathrm{p} z}^{+} & =-\frac{2 u_{\mathrm{p}}^{-}}{D_{\mathrm{p}}}
\end{aligned}
$$

If the condition (52) is not fulfilled, a sliding collision of a spherical particle with the wall is calculated with the equations:

$$
\begin{aligned}
u_{\mathrm{p}}^{+} & =u_{\mathrm{p}}^{-}+\varepsilon_{x} \mu_{\mathrm{w}}\left(1+e_{\mathrm{w}}\right) v_{\mathrm{p}}^{-} \\
v_{\mathrm{p}}^{+} & =-e_{\mathrm{w}} v_{\mathrm{p}}^{-} \\
w_{\mathrm{p}}^{+} & =w_{\mathrm{p}}^{-}+\varepsilon_{z} \mu_{\mathrm{w}}\left(1+e_{\mathrm{w}}\right) v_{\mathrm{p}}^{-} \\
\omega_{\mathrm{p} x}^{+} & =\omega_{\mathrm{p} x}^{-}-5 \varepsilon_{z} \mu_{\mathrm{w}}\left(1+e_{\mathrm{w}}\right) \frac{v_{\mathrm{p}}^{-}}{D_{\mathrm{p}}} \\
\omega_{\mathrm{p} y}^{+} & =\omega_{\mathrm{py}}^{-} \\
\omega_{\mathrm{p} z}^{+} & =\omega_{\mathrm{p} z}^{-}+5 \varepsilon_{x} \mu_{\mathrm{w}}\left(1+e_{\mathrm{w}}\right) \frac{v_{\mathrm{p}}^{-}}{D_{\mathrm{p}}}
\end{aligned}
$$

where $\varepsilon_{x}$ and $\varepsilon_{z}$ are:

$\varepsilon_{x}=\left(u_{\mathrm{p}}^{-}+\frac{D_{\mathrm{p}}}{2} \omega_{\mathrm{p} z}^{-}\right) /|\mathbf{U}|$

$\varepsilon_{z}=\left(w_{\mathrm{p}}^{-}-\frac{D_{\mathrm{p}}}{2} \omega_{\mathrm{p} x}^{-}\right) /|\mathbf{U}|$

In the application of the $2 \mathrm{D}$ rough wall multiple-collision procedure, the particle impact with the wall is calculated according to the procedure outlined by Konan et al. (2009).

As can be seen from Fig. 16, in the $x-y$ plane, the agreement between the experimental results, the $2 \mathrm{D}$ rough wall multiplecollision model and the 3D stochastic procedure is very good for the incident angles $\alpha_{\mathrm{p}}^{-}=-5^{\circ}, \alpha_{\mathrm{p}}^{-}=-15^{\circ}$ and $\alpha_{\mathrm{p}}^{-}=-25^{\circ}$.

Although friction is accounted for in particle-wall collisions, the distribution of particle rebound angles $\beta_{\mathrm{p}}^{+}$is still Gaussian, as in the case of fully elastic particle rebounds, with zero mean value and a standard deviation of the rebound angle $\beta_{\mathrm{p}}^{+}$that increases with the amplitude of the incident angle $\left|\alpha_{\mathrm{p}}^{-}\right|$, as can be seen in Fig. 16(b), (d) and (f).

\subsection{Dependence of the transverse bouncing velocity standard deviation on the wall materials and particle diameter from deterministic and stochastic simulations}

Fig. 17 shows the dependence of the ratio of transverse velocity standard deviation to particle mean incident velocity magnitude $\sigma_{\mathrm{p}}^{+} /\left|\mathbf{U}_{\mathrm{p}}^{-}\right|$on the ratio of particle incident angle to virtual normal vector angle standard deviation $\left|\alpha_{\mathrm{p}}^{-}\right| / \Delta \xi$ using deterministic and stochastic simulations carried out using the experimental parameters from Sommerfeld and Huber (1999) for various wall materials and glass particles, for a particle transverse incident angle $\beta_{\mathrm{p}}^{-}=0^{\circ}$. However, considering that the simulated virtual wall is isotropic, the results obtained are independent of the particle incident angle $\beta_{\mathrm{p}}^{-}$. 
In all simulated cases, the angular velocity of particles is the same as in Section 5.1. Simulated incident particle angles are up to around $40^{\circ}$, since in the original experiment the channel was narrow and the particle incident angles were small.

Among the available wall materials and sizes of particles, the largest transverse particle dispersion is observed for a steel wall and glass particles with diameter $D_{\mathrm{p}}=100 \mu \mathrm{m}$ (equivalent to an isotropic wall with virtual normal angle standard deviation $\Delta \xi=$ $\Delta \zeta=5.3^{\circ}$ ). For this configuration, at the largest particle incident angles the generated transverse velocity standard deviation $\sigma_{\mathrm{p}}^{+}$ is around $8 \%$ of the inlet velocity. The lowest transverse particle dispersion is for a polished steel wall and particle diameter $D_{\mathrm{p}}=100 \mu \mathrm{m}$ (equivalent to an isotropic wall with virtual normal angle standard deviation $\Delta \xi=\Delta \zeta=2.3^{\circ}$ ): at the largest particle incident angles, the standard deviation of the generated transverse velocity $\sigma_{\mathrm{p}}^{+}$is around $4 \%$ of the inlet velocity.

A steel wall and particle of diameter $D_{\mathrm{p}}=500 \mu \mathrm{m}$ and a plexiglass wall and particle of diameter $D_{\mathrm{p}}=100 \mu \mathrm{m}$, both configurations with normal vector angle standard deviation $\Delta \xi=\Delta \zeta=$ $3.8^{\circ}$, have approximately equal transverse particle dispersions. Although a rubber wall and particles of diameter $D_{\mathrm{p}}=100 \mu \mathrm{m}$ have a normal vector angle standard deviation $\Delta \xi=\Delta \zeta=3.8^{\circ}$, the transverse particle dispersion is slightly lower than in the previous two configurations with the same virtual normal angle standard deviations.

Overall, in the simulated cases, the agreement between the deterministic simulation results and the stochastic model predictions is excellent.

\section{Conclusion}

In this paper, a new stochastic procedure for 3D particle-rough wall interaction in a Lagrangian framework has been developed. This procedure represents an extension of the models developed by Sommerfeld and Huber (1999) and Konan et al. (2009), both of which account for the particle-wall interaction by modelled 2D wall roughness structures. Compared with these models, considering that the impact of a particle with a wall is generally $3 \mathrm{D}$, the new stochastic procedure predicts a more realistic particle rebound with a transverse particle bouncing velocity component.

Deterministic simulations using isotropic random rough surfaces generated by following Garcia and Stoll (1984) show that the PDFs of the first virtual normal vector angles $\xi$ and $\zeta$ seen by incident particles are conditioned by the particle incident angle $\alpha_{\mathrm{p}}^{-}$, in agreement with the shadow effect characterized by Sommerfeld and Huber (1999) in the frame of the 2D rough wall modelling approach. Also, it is shown that the PDF of the rebound angle $\alpha_{\mathrm{p}}^{+}$agrees very well with the that predicted by the stochastic model of Konan et al. (2009), which accounts for the shadow effect and multiple particle-wall collisions. Therefore, to allow the link with this model, a unit vector $\mathbf{n}_{\gamma}$ in the particle incident plane is introduced. This vector is given by assuming that its scalar projection onto the particle incident velocity $\mathbf{U}_{\mathrm{p}}^{-}$is equal to that of the wall normal unit vector $\mathbf{n}$. To account for the transverse deviation bouncing effect, the angle $\gamma^{*}$ obeying a given Gaussian PDF is introduced. It is shown that the angle $\gamma^{*}$ is not affected by the shadow effect and multiple particle-wall collisions. The stochastic procedure coupling $\gamma$ and $\gamma^{*}$, accounting for the transverse bouncing effect, is then derived.

Owing to the 3D roughness effect, the particle bouncing velocities show a transverse random component with standard deviation proportional to the sine of the incident angle amplitude $\left|\alpha_{\mathrm{p}}^{-}\right|$and to the standard deviations of the virtual wall normal vector angles $\Delta \xi=\Delta \zeta$.

In further studies, using Euler-Lagrangian simulations of particle-laden flows, comparisons between different stochastic pro- cedures for particle-wall interactions and experimental results should be made in different flow configurations. The application of the new stochastic procedure is expected to give more accurate results in particle-laden flows where the influence of wall roughness is important, especially in flows with emphasized 3D particle movement and with large particle incident angles $\alpha_{\mathrm{p}}^{-}\left(\left|\alpha_{\mathrm{p}}^{-}\right| \gg\right.$ $\Delta \xi$ and $\Delta \zeta$ ). Also, since anisotropic surfaces are often encountered in engineering practice, the interaction between particles and anisotropic rough walls will be investigated further.

\section{Acknowledgements}

The research of the first author was financially supported by the Ministry of Education, Science and Technological Development of the Republic of Serbia, project no. TR 35046, and is gratefully acknowledged.

\section{References}

Benson, M., Tanaka, T., Eaton, J., 2005. Effects of wall roughness on particle velocities in a turbulent channel flow. ASME J. Fluids Eng. 127, 250-256. doi:10.1015/ 1.1891149.

Bergström, D., 2012. MySimLabs. http://www.mysimlabs.com/surface_generation. html. Accessed: 2 Feb. 2017.

Breuer, M., Alletto, M., Langfeldt, F., 2012. Sandgrain roughness model for rough walls within Eulerian-Lagrangian predictions of turbulent flows. Int. J. Multiphase Flow 43, 157-175.

Cheng, Z., Zhu, M., 2015. Analyzing the effect of wall roughness on gas-particle flow in confined channels based on a virtual-wall-group concept. Int. J. Multiphase Flow 77, 158-170.

De Marchis, M., Milici, B., Sardina, G., Napoli, E., 2016. Interaction between turbulent structures and particles in roughened channel. Int. J. Multiphase Flow 78, 117-131.

Garcia, N., Stoll, E., 1984. Monte Carlo calculation for electromagnetic-wave scattering from random rough surfaces. Phys. Rev. Lett. 52, 1798-1801.

Konan, A., Badarayani, S., Simonin, O., Squires, K., 2007. LES/DPS of horizontal gassolid channel flow with particle-particle collision and wall roughness effects. In: 6th International Conference on Multiphase Flow. ICMF2007, Leipzig, Germany, p. 11.

Konan, N.A., Kannengieser, O., Simonin, O., 2009. Stochastic modeling of the multiple rebound effects for particle-rough wall collisions. Int. J. Multiphase Flow 35 933-945. doi:10.1016/j.ijmultiphaseflow.2009.05.006

Konan, N.A., Simonin, O., Squires, K.D., 2011. Detached eddy simulations and particle Lagrangian tracking of horizontal rough wall turbulent channel flow. J. Turbul. $12,1-21$.

Malloupas, G., van Wachem, B., 2013. Large eddy simulation of turbulent particle-laden channel flow. Int. J. Multiphase Flow 54, 65-75.

Milici, B., 2018. Modification of particle laden near-wall turbulence in a vertical channel bounded by rough walls. Int. J. Multiphase Flow 103, 151-168.

Sommerfeld, M., 2003. Analysis of collision effects for turbulent gas-particle flow in a horizontal channel: part I. Particle transport. Int. J. Multiphase Flow 29, 675-699.

Sommerfeld, M., Huber, N., 1992. Modelling of particle-wall collisions in confined gas-particle flows. Int. J. Multiphase Flow 18, 905-926.

Sommerfeld, M., Huber, N., 1995. A laser strobe technique combined with digital image analysis to study particle-wall collisions. In: Modern Measuring Techniques for Multiphase flows. Proceedings of the International Symposium on Measurement Techniques for Multiphase flows, Nanjing, China, p. 13.

Sommerfeld, M., Huber, N., 1999. Experimental analysis and modelling of particle-wall collisions. Int. J. Multiphase Flow 25, 1457-1489.

Sommerfeld, M., Kussin, J., 2004. Wall roughness effects on pneumatic conveying of spherical particles in a narrow horizontal channel. Powder Tech. 142, 180-192. doi:10.1016/j.powtec.2004.05.002.

Squires, K., Simonin, O., 2006. LES-DPS of the effect of wall roughness on dispersedphase transport in particle-laden turbulent channel flow. Int. J. Heat Fluid Flow 29, 619-629. doi:10.1016/j.ijheatfluidflow.2006.02.009.

Tsang, L., King, J.A., Ding, K.-H., 2000. Scattering of Electromagnetic Waves: Theories and Applications. John Wiley and Sons, New York.

Tsuji, Y., Morikawa, Y., Tanaka, T., Nakatsukasa, N., Nakatani, M., 1987. Numerical simulation of gas-solid two-phase flow in a two-dimensional horizontal channel. Int. J. Multiphase Flow 13 (5), 671-684.

Tsuji, Y., Oshima, T., Morikawa, Y., 1985. Numerical simulation of pneumatic conveying in a horizontal pipe. KONA 3, 38-51.

Vreman, A., 2007. Turbulence characteristics of particle-laden pipe flow. J. Fluid Mech. 584, 235-279.

Vreman, A., 2015. Turbulence attenuation in particle-laden flow in smooth and rough channels. J. Fluid Mech. 773, 103-136. 